\title{
Long-Run Money Demand Function and Stability among Sixteen (16) West Africa Countries
}

\author{
Michael Asiedu ${ }^{1 *}$, Pious Opoku², Anwar Mohsen AbdElghaffar ${ }^{3}$, \\ Patrick Bimpong ${ }^{4}$, Frank Yeboah ${ }^{5}$
}

\author{
${ }^{1}$ School of Finance, Zhongnan University of Economics and Law, Wuhan, China \\ ${ }^{2}$ School of Accounting and Finance, Zhongnan University of Economics and Law, Wuhan, China \\ ${ }^{3}$ Department of Accounting and Auditing, Assuit University, Assuit, Egypt \\ ${ }^{4}$ School of Accounting and Finance, Zhongnan University of Economics and Law, Wuhan, China \\ ${ }^{5}$ Department of Accounting and Finance, Kwame Nkrumah University of Science and Technology, Kumasi, Ghana \\ Email: ^masiedu256@gmail.com,piousopoku1990@gmail.com,mohsen333@aun.edu.eg, \\ pbimpong87@gmail.com,frank.yeboah98@gmail.com
}

How to cite this paper: Asiedu, M., Opoku, P., AbdElghaffar, A. M., Bimpong, P., \& Yeboah, F. (2020). Long-Run Money Demand Function and Stability among Sixteen (16) West Africa Countries. Journal of Financial Risk Management, 9, 390-417. https://doi.org/10.4236/jfrm.2020.94021

Received: September 9, 2020

Accepted: November 3, 2020

Published: November 6, 2020

Copyright $\odot 2020$ by author(s) and Scientific Research Publishing Inc. This work is licensed under the Creative Commons Attribution International License (CC BY 4.0).

http://creativecommons.org/licenses/by/4.0/

\begin{abstract}
This study employs annual time series data from 1982 to 2019 for sixteen (16) West Africa countries to investigate the stability of money demand in these countries and the feasibility of the proposed West African Monetary Zone. From a standard money demand function to a bounds test method, co-integration and error correction model, we found significant heterogeneity and divergence across the sixteen (16) countries. The highlight of the findings is that co-integration of the money demand function is only recorded in Ghana but exhibited partial stability from the CUSUMSQ (cumulative sum squared) test. In Nigeria, the biggest economy in West Africa has no co-integration and is only partially stable from the results of the CUSUM test. The significant divergence across these countries as indicated by the test of co-integration, CUSUM (cumulative sum) test, CUSUMSQ (cumulative sum squared) test, the short and long-run factors and error correction in the event of a shock, makes it crucial for the need to include country specific idiosyncratic monetary policy features in the event of a monetary zone in West Africa, and crucial to ensure convergence in their money demand function.
\end{abstract}

\section{Keywords}

Money Demand, Stability, Co-Integration, ARDL Test, CUSUM and CUSUMSQ

\section{Introduction}

The factors underlining money demand function and its long-run stability have 
gained much attention among economist with particular attention to how foreign factors and exchange rate fluctuations affect the stability of a domestic currency. Out of the concept of creating a monetary zone in West Africa and the adoption of one currency for all 16 countries is the West Africa Monetary Zone (WAMZ). A monetary zone or a common currency area is defined as an economic area within which exchange rates are fixed. Even though some countries within West Africa (termed as Francophone) countries have the same currency (CFA) as medium of exchange, there are still many variations in their respective levels of monetary aggregates. Countries in West Africa have not experience major financial crisis; yet have suffered significantly from the Asia Financial Crises of 1997 and the global financial crises of 2008 which had its roots from US due to complex financial algorithms. Volatility in external factors significantly affects the fragile economies of these countries. These factors such as changes in international interest rate and exchange rate have significant effects on domestic currency holdings and asset allocations.

For most Central Banks in West Africa, they are tasked with the main goal of achieving and maintaining; growth and price stability both in the medium and long-term. In pursuant to these goals, central banks are guided by rigorous analysis of the monetary aggregates in their economies and sometimes the cross country or international effects of their actions. The analysis of monetary aggregates is considered a major task of central banks because of the significant roles of money playing in achieving both the medium and long-term goals of these central banks. Many studies have been conducted in Africa on money demand functions and stability; the feasibility of a currency area in West Africa and other African sub-regional blocks due to the need for monetary aggregates measures that are theoretically consistent for economic unions, such as the proposed single currency for the West Africa Monetary Zone, is highly relevant. Baharumshah et al. (2009) examined the demand for M2 in China using the bounds test through the autoregressive distributed lag and co-integration framework to establish a stable and long-run relationship between M2 and the independent variables (determinants); real income, foreign interest rate, inflation and stock prices. Specifically, they found that the omission of stock prices can result in model misspecification and misleading results in the demand for money function; this is because the wealth effects of stock prices were found to be significant on the long and short-run broad money demand. This key finding is not at variance with the school of thought that holds that asset inflation exerts some effect on the level of monetary aggregates. In a related study on the long-run demand for money by Bahmani-Oskooee \& Chi (2002) in Hong Kong, they found that M2 (HK\$M2) has long-run relationship with its determinants using data from the first quarter of 1985 to the final quarter of 1999. They also confirmed stability in the money demand function from both the results of the CUSUM and CUSUMSQ test. Asongu et al. (2019b) in their study on the stability of money demand for thirteen countries in West African as part of their fea- 
sibility study of the West African Monetary Union (WAMU), employed annual data from 1981 to 2015, and they found significant divergence across these countries in the stability of demand for money. The divergence they recorded was due to difference in long-run relationship (co-integration), short and long-run factors, stability, and error correction in the event of any shock. Harvey \& Cushing (2015) in their quest to establish the presence of a common currency area in the West African Monetary Zone (WAMZ) also adopted a structural autoregressive model to understand the variance decomposition and impulse response functions. They found that these countries responded asymmetrically to systematic supply and demand for money shocks following structural shocks and therefore reacted idiosyncratically to a universal or systematic monetary policy. From the variance decomposition, they also found that the West African Monetary Zone (WAMZ) as a whole did not exhibit a systematic source of shock principally due to the variations and diversity in the structures of their economies. They therefore concluded that unless there is a convergence of the economies of these countries; they should not go into a monetary union. Diop et al. (2017) used monetary stress indicators obtained from backward-looking Taylor rule to assess the convergence of macroeconomic variables in the future of West Africa monetary union; established a significant drop of monetary stress since the coming into force the convergence pact. They also found a decrease in stress following the decompositioning of monetary stress due to varying rates of inflation since 1999 with observed asymmetries as a result of cyclical output among ECOWAS countries. In their conclusion, they observed that monetary policy may be too accommodative or too tight for NON-WAEMU and WAEMU countries respectively. This implies that the target rate of future central banks will not meet the aspirations of every member country.

Hossain (1993) also estimated a short run money demand model and test for stability in Bangladesh using quarterly data from the 1974 to 1989 . The results from the Chow, CUSUM and CUSUMSQ revealed that broad money demand for the most part of the 1980s was stable however; narrow money was unstable during the period 1982 to 1987 . He therefore concluded that, the instability recorded must be as a result of financial sector reforms in the 1980s in Bangladesh. In a related study conducted by Rao \& Kumar (2009) on financial reforms and demand for money using panel data of fourteen (14) countries in Asia from 1970 to 2005 ; through co-integrating equations, they found the money demand function to be stable but the significant effects of financial reforms were yet to manifest. In the absence of any trace of instability in the demand for money function, they recommended the choice of money supply over interest rate as the main policy tool. Kumar (2011) analysed data from twenty (20) Asia and African countries and found from the CUSUM and CUSMUSQ that the demand for money functions are stable and therefore recommended to monetary authorities to use money supply as their key target. Folarin \& Asongu (2017) were of the conviction that interest rate is an ineffective monetary policy tool in Nigeria. 
This conclusion was made after they conducted an examination of the stability of money demand function in Nigeria post financial sector reforms. The results from the CUSUM and CUSUMSQ and the ARDL bounds test found the existence of co-integration (long-run relationship) among the variables and stability in the demand for money function in Nigeria. The results from an asymmetric co-integration and error-correction model conducted in Malaysia by Roohollah \& Azali (2015) with monthly data from 1986 to 2012 on the relationship between stock prices and monetary policy found evidence of co-integration between monetary aggregates and stock prices except finance, consumer products and plantation sectors. In addition; they found that monetary policy is asymmetrically co-integrated with industrial and properties stock price indices. Cassola \& Morana (2004) in their study evaluating the mutual consistency and complementarity of price stability and financial stability objectives in the Euro area concluded that: 1) stock market stability can be achieved in the long-run when monetary policy is targeted at price stability; 2) the driving factor of the stock market in the long-run is permanent productivity shocks; 3 ) they did not establish significant and direct effect of stock prices on inflation; 4) in the Euro area, asset prices (stock prices) were found to be significantly important in the monetary transmission mechanism. James (2005) adopted a strategy that explicitly incorporates the effects of financial liberalization in the money demand function as an alternative to the bounds and standard co-integration test for his study. $\mathrm{He}$ found that in Indonesia, financial liberalization is a significant factor influencing the stability of demand for money. In testing for the stability of the money demand function in Japan, Hamori \& Tokihisa (2001) employed the notion of a seasonal co-integration in their empirical analysis. A unit root process in different periods was found in money balances, interest rates and real income. However, in every case the results from the seasonal co-integration were rejected. They therefore concluded that there is no evidence of a stable relationship between the real economy and demand for money for the period under consideration. Foresti \& Napolitano (2014) in their study investigating which monetary aggregate influences the stability of money demand within the European Monetary Union (EMU) employed panel data estimation technique and found that the relationship between money demand stability and the determinants change based on the monetary aggregate used as proxy for money demand. They found significant stability in money demand when M2 is adopted. However, the money demand stability significantly improves when M2 is substituted for M3. Their result was also robust after dividing the sample into two unique groups. They found that the inflationary effect on money demand is very high for economies that are unstable while income plays a more significant role in economies that are stable. Debrun \& Masson (2013) from their study on Common Monetary Area (CMA) among Southern African Development Community (SADC) found that member countries will benefit substantially except Mauritius. From a structural vector auto-regression Darvas (2015) created an Euro-area Divisia-money 
dataset and estimated a theoretically right response to demand for money, interest rate shocks and user fees. They concluded that money plays a significant role for output, price levels and interest rates and monetary developments can be achieved through the European Central Bank. Boone \& Van den Noord (2004) also investigated the factors influencing money demand (M3) within the Euro area particularly on the effect of financial and housing wealth on money demand. They found evidence of a positive long-run wealth effect from the dependent variables on money demand, but no evidence of short-run effect was recorded. Part of their findings also confirms the stability of the long-run and dynamic money demand equation; and the introduction of the euro in January 1999 did not disrupt it. From a system dynamic model Fleissig \& Swofford (1996) estimated equations of money demand and found substitutability among monetary variables such as cash, savings and time deposits. From a recent study by Dritsaki \& Dritsaki (2020) who examined the determinants and the stability of money demand function in Italy using annual time series data from 1960 to 2017 using an autoregressive distributed lag (ARDL) and vector error correction techniques, they established the presence of long-run and short run relationship between money demand and its determinants. The stability of the parameters was also confirmed by the CUSUM test for stability in Italy using narrow money (M1) as the baseline monetary aggregate. By implication, monetary policymakers are advised to moderately target broad money aggregates with all these asset classes to achieve maximum and reliable effect.

\section{Data Source and Scope}

The data for the study is annual data secured from the World Development Indicators website for all the sixteen (16) countries in West Africa spanning from 1982 to 2019 except for the LIBOR data which was sourced from Macrotrends.net.

\section{Definition of Variables and Justification}

The variables adopted in this study are based on a careful review of the literature following Asongu et al. (2019a) on the stability of demand for money in the proposed Southern Africa Monetary Union. Real Broad Money (RM2) is the expression for nominal broad money divided by the GDP deflator. Inflation is the percentage change in the annual GDP deflator. Exchange rate is the official exchange rate of the local currency expressed in terms of the US dollar. Real income (real GDP) is also the annual nominal GDP divided by the GDP deflator. LIBOR rate is the three-month London Inter-Bank Overnight rate. The relevance of the foreign interest rate (LIBOR) is to capture the substitution effect between domestic assets and foreign interest-bearing assets as indicated by Debrun \& Masson (2013). The choice of variables are extensively justified and reviewed in the literature by Bahmani-Oskooee \& Gelan (2009), Asongu et al. (2019a) and Asongu et al. (2019b). 


\section{Methodology}

Based on the relevant literature on the stability of money demand function and co-integration method (Bahmani-Oskooee \& Gelan, 2009; Asongu et al., 2019a); the Auto-regressive Distributed lag model (ARDL) is adopted due to its consistent features with the data. The ARDL's appropriateness is based on the fact that it ideal when the series used are made of variables integrated of order zero I(0) and order I(1). To test for the stability of the money demand functions; CUSUM test and CUSUM square test of Brown et al. (1975) are also deployed as in the case of Kumar (2011) for twenty (20) Asian and African countries.

Model specification

$$
\mathrm{RM} 2=f(\text { Exch, Infl, RGDP, Libor })
$$

where RM2 is real monetary aggregate (M2), Exch is the real official effective exchange rate, Libor is the representation of the foreign interest rate, RGDP is real income.

Equation (1) is transformed into Equation (2)

$$
\ln \mathrm{RM}_{t}=\beta_{0}+\beta_{1} \text { Exch }_{t}+\beta_{2} \operatorname{Infl}_{t}+\beta_{3} \ln _{\mathrm{RGDP}_{t}}+\beta_{4} \text { Libor }_{t}+\varepsilon_{t}
$$

ARDL model specification

The equation for the auto-regressive distributed lag (ARDL) model is specified as below after the test for unit root I(0), I(1), co-integration and bounds test.

$$
\begin{aligned}
\Delta(\ln \mathrm{RM} 2)_{t}= & \lambda_{0}+\lambda_{1}(\ln \mathrm{RM} 2)_{t-1}+\lambda_{2} \operatorname{Exch}_{t-1}+\lambda_{3} \operatorname{Infl}_{t-1}+\lambda_{4} \ln \mathrm{RGDP}_{t-1} \\
& +\lambda_{5} \operatorname{Libor}_{t-1}+\sum_{j=1}^{l} \mathcal{I} 1 j \Delta(\ln \mathrm{RM} 2)_{t-j} \\
& +\sum_{j=0}^{m} \mathcal{I} 2 j \Delta \ln \operatorname{RGDP}_{t-j}+\sum_{j=0}^{n} \mathcal{I} 3 j \Delta \operatorname{Exch}_{t-j} \\
& +\sum_{j=0}^{o} \mathcal{I} 4 j \Delta \operatorname{Infl}_{t-j}+\sum_{j=0}^{p} \mathcal{I} 5 j \Delta \operatorname{LIBOR}_{t-j}+\varepsilon_{t}
\end{aligned}
$$

From the evidence of one co-integration (Ghana) the Error Correction Model is specified

$$
\mathrm{ECT}=\ln (\mathrm{RM} 2)_{t}-\left(\delta_{0}+\delta_{1} \mathrm{Exch}_{t}+\delta_{2} \mathrm{Infl}_{t}+\delta_{3} \ln \mathrm{RGDP}_{t}+\delta_{4} \text { Libor }_{t}\right)
$$

\subsection{Summary Statistics}

All Sixteen (16) West African countries

Table 1. Descriptive statistics.

\begin{tabular}{cccccc}
\hline Variable & Obs & Mean & Std.Dev. & Min & Max \\
\hline lnRM2 & 608 & -0.117 & 88.956 & -1487.562 & 558.385 \\
Infl & 608 & 11.473 & 19.661 & -29.172 & 165.677 \\
lnRGDP & 608 & -0.054 & 95.474 & -1625.81 & 617.215 \\
Exch & 608 & 610.363 & 1301.641 & 0 & 9183.876 \\
LIBOR & 608 & 4.33 & 3.067 & 0.23 & 9.73 \\
\hline
\end{tabular}

French Speaking countries 
Table 2. Descriptive statistics.

\begin{tabular}{cccccc}
\hline Variable & Obs & Mean & Std.Dev. & Min & Max \\
\hline lnRM2 & 342 & -0.216 & 117.327 & -1487.562 & 558.385 \\
Infl & 342 & 5.833 & 10.149 & -11.876 & 100.627 \\
InRGDP & 342 & -0.246 & 126.085 & -1625.81 & 617.215 \\
Exch & 342 & 732.756 & 1351.386 & 5.177 & 9183.876 \\
LIBOR & 342 & 4.33 & 3.069 & 0.23 & 9.73 \\
\hline
\end{tabular}

Others (English and Portuguese Speaking countries)

Table 3. Descriptive statistics.

\begin{tabular}{cccccc}
\hline Variable & Obs & Mean & Std.Dev. & Min & Max \\
\hline InRM2 & 266 & 0.011 & 20.306 & -176.766 & 50.353 \\
Infl & 266 & 18.724 & 25.672 & -29.172 & 165.677 \\
lnRGDP & 266 & 0.192 & 20.547 & -180.262 & 50.671 \\
Exch & 266 & 452.999 & 1219.281 & 0 & 9010.221 \\
LIBOR & 266 & 4.33 & 3.071 & 0.23 & 9.73 \\
\hline
\end{tabular}

Tables 1-3 report the descriptive statistics of the all the countries, French speaking countries and the Others (English and Portuguese Speaking countries) respectively. The report indicates significant dispersions from their mean values.

\subsection{Correlation Matrix}

For the entire sample

Table 4. Pairwise correlations

\begin{tabular}{lccccc}
\hline Variables & $(1)$ & $(2)$ & $(3)$ & $(4)$ & $(5)$ \\
\hline (1) $\ln R M 2$ & 1.000 & & & & \\
(2) Infl & 0.015 & 1.000 & & & \\
(3) $\operatorname{lnRGDP}$ & $1.00^{*}$ & 0.015 & 1.000 & & \\
(4) Exch & 0.022 & -0.06 & 0.022 & 1.000 & \\
(5) LIBOR & -0.01 & $0.23^{*}$ & -0.02 & $-0.26^{*}$ & 1.000
\end{tabular}

*Shows significance at the 0.05 level.

For French speaking countries

Table 5. Pairwise correlations

\begin{tabular}{lccccc}
\hline Variables & $(1)$ & $(2)$ & $(3)$ & (4) & (5) \\
\hline (1) $\ln R M 2$ & 1.000 & & & & \\
$(2)$ Infl & 0.007 & 1.000 & & \\
(3) $\operatorname{lnRGDP}$ & $1.00^{*}$ & 0.006 & 1.000 \\
\hline
\end{tabular}




\section{Continued}

\begin{tabular}{llllll}
\hline (4) Exch & 0.016 & $0.14^{*}$ & 0.016 & 1.000 & \\
(5) LIBOR & -0.09 & $0.15^{*}$ & -0.09 & $-0.28^{*}$ & 1.000 \\
\hline
\end{tabular}

*Shows significance at the 0.05 level.

For English speaking countries

Table 6. Pairwise correlations.

\begin{tabular}{lccccc}
\hline Variables & $(1)$ & $(2)$ & $(3)$ & $(4)$ & (5) \\
\hline (1) $\operatorname{lnRM} 2$ & 1.000 & & & & \\
(2) Infl & 0.051 & 1.000 & & & \\
(3) $\operatorname{lnRGDP}$ & $1.00^{*}$ & 0.050 & 1.000 & & \\
(4) Exch & 0.047 & -0.08 & 0.048 & 1.000 & \\
(5) LIBOR & 0.065 & $0.34^{*}$ & 0.066 & $-0.26^{*}$ & 1.000 \\
\hline
\end{tabular}

*Shows significance at the 0.05 level.

Tables 4-6 report the correlation matrix of the all the countries French speaking countries and the Others (English and Portuguese Speaking countries) respectively. The report indicates that the correlation between the variables is not strong enough to pose the challenge of multi-collinearity.

Table 7. Panel unit root test.

\begin{tabular}{|c|c|}
\hline Variable & $P$-Value \\
\hline Infl (level) & $0.0000^{* * *}$ \\
\hline Infl ( $1^{\text {st }}$ diff $)$ & $0.0000^{* * *}$ \\
\hline LnRM2 (level) & $0.0000^{* * *}$ \\
\hline LnRM2 ( $1^{\text {st }}$ diff) & $0.0000^{* * *}$ \\
\hline LnRGDP (level) & $0.0000^{* * *}$ \\
\hline $\operatorname{LnRGDP}\left(1^{\text {st }}\right.$ diff $)$ & $0.0000^{* * *}$ \\
\hline Exch (level) & 1.0000 \\
\hline $\operatorname{Exch}\left(1^{\text {st }}\right.$ diff $)$ & $0.0000^{* * *}$ \\
\hline LIBOR (level) & $0.0001^{* * *}$ \\
\hline LIBOR ( $1^{\text {st }}$ diff $)$ & $0.0000^{* * *}$ \\
\hline
\end{tabular}

${ }^{* * *}$ Shows significance at the 0.01 level

Table 7 above, is also the panel unit root test of the variables. As indicated by the probability values ( $P$-value) all the panel variables are significant at level at $1 \%$ significance level indicating the absence of unit root except exchange rate which is significant after first difference.

The unit root test reported in Table 8 through the probability values indicates that except the LIBOR rate most of the variables are stationary at their level. The LIBOR rate is stationary after the first difference. 
Table 8. Unit root test for country level.

\begin{tabular}{|c|c|c|c|c|c|c|c|c|}
\hline \multirow{3}{*}{ Variable } & \multicolumn{8}{|c|}{ COUNTRY } \\
\hline & Senegal & Sierra L. & TOGO & Nigeria & Niger & Mali & Mauritania & Liberia \\
\hline & \multicolumn{8}{|c|}{$P$-Value } \\
\hline Infl (level) & $0.0029^{* * *}$ & 0.2391 & $0.0003^{* * *}$ & $0.0111^{* *}$ & $0.0000^{\star * *}$ & $0.0003^{* * *}$ & $0.0019^{* * *}$ & $0.0051^{* * *}$ \\
\hline Infl ( $1^{\text {st }}$ diff $)$ & $0.0000^{* * *}$ & $0.0000^{\star * *}$ & $0.0000^{* * *}$ & $0.0000^{* * *}$ & $0.0000^{\star * *}$ & $0.0000^{\star * *}$ & $0.0000^{* * *}$ & $0.0000^{* * *}$ \\
\hline lnRM2 (level) & $0.0003^{* * *}$ & $0.0383^{\star *}$ & $0.0000^{\star * *}$ & $0.0018^{\star * *}$ & $0.0006^{* * *}$ & $0.0043^{* * *}$ & $0.0009^{* * *}$ & $0.0011^{* * *}$ \\
\hline $\operatorname{lnRM} 2\left(1^{\text {st }}\right.$ diff $)$ & $0.0000^{* * *}$ & $0.0000^{* * *}$ & $0.0000^{* * *}$ & $0.0000^{* * *}$ & $0.0000^{* * *}$ & $0.0000^{* * *}$ & $0.0000^{* * *}$ & $0.0000^{\star * *}$ \\
\hline lnRGDP (level) & $0.0003^{* * *}$ & $0.0259^{* *}$ & $0.0000^{\star * *}$ & $0.0017^{\star * *}$ & $0.0006^{\star * *}$ & $0.0040^{\star * *}$ & $0.0009^{\star * *}$ & $0.0010^{* * *}$ \\
\hline $\operatorname{lnRGDP}\left(1^{\text {st }}\right.$ diff $)$ & $0.0000^{* * *}$ & $0.0000^{\star * *}$ & $0.0000^{* * *}$ & $0.0000^{* * *}$ & $0.0000^{\star * *}$ & $0.0000^{* * *}$ & $0.0000^{* * *}$ & $0.0000^{* * *}$ \\
\hline Exch (level) & 0.4522 & 0.9991 & 0.4521 & 0.9828 & 0.4521 & 0.4521 & 0.9395 & 1.0000 \\
\hline Exch ( $1^{\text {st }}$ diff $)$ & $0.0002^{* * *}$ & 0.3820 & $0.0002^{* * *}$ & $0.0050^{\star * *}$ & $0.0002^{* * *}$ & $0.0002^{\star * *}$ & $0.0042^{\star * *}$ & 0.9969 \\
\hline LIBOR (level) & 0.1602 & 0.1602 & 0.1602 & 0.1602 & 0.1602 & 0.1602 & 0.1602 & 0.1602 \\
\hline LIBOR ( $1^{\text {st }}$ diff $)$ & $0.0000^{* * *}$ & $0.0000^{* * *}$ & $0.0000^{* * *}$ & $0.0000^{\star * *}$ & $0.0000^{* * *}$ & $0.0000^{\star * *}$ & $0.0000^{\star * *}$ & $0.0000^{* * *}$ \\
\hline Variable & Ivory Coast & Guinea Bissau & Guinea & Ghana & Gambia & Cape Verde & Burkina Faso & Benin \\
\hline Infl (level) & $0.0010^{* * *}$ & 0.4999 & 0.0411 & $0.0000^{* * *}$ & $0.0009^{* * *}$ & $0.0000^{\star * *}$ & $0.0002^{\star * *}$ & $0.0029^{* * *}$ \\
\hline Infl ( $1^{\text {st }}$ diff $)$ & $0.0000^{\star * *}$ & $0.0000^{\star * *}$ & $0.0000^{\star * *}$ & $0.0000^{* * *}$ & $0.0000^{* * *}$ & $0.0000^{* * *}$ & $0.0000^{\star * *}$ & $0.0000^{* * *}$ \\
\hline lnRM2 (level) & $0.0007^{\star \star *}$ & $0.0055^{\star * *}$ & $0.0008^{\star * *}$ & 0.6350 & $0.0024^{\star * *}$ & $0.0302^{\star *}$ & $0.0001^{\star * *}$ & $0.0003^{* * *}$ \\
\hline $\operatorname{lnRM} 2\left(1^{\text {st }}\right.$ diff $)$ & $0.0000^{\star * *}$ & $0.0000^{\star * *}$ & $0.0000^{\star * *}$ & $0.0000^{* * *}$ & $0.0000^{\star * *}$ & $0.0000^{\star * *}$ & $0.0000^{* * *}$ & $0.0000^{* * *}$ \\
\hline lnRGDP (level) & $0.0007^{\star * *}$ & $0.0056^{* * *}$ & $0.0009^{* * *}$ & 0.6003 & $0.0021^{\star * *}$ & $0.0305^{\star *}$ & $0.0001^{* * *}$ & $0.0003^{* * *}$ \\
\hline $\operatorname{lnRGDP}\left(1^{\text {st }}\right.$ diff $)$ & $0.0000^{\star * *}$ & $0.0000^{\star * *}$ & $0.0000^{\star * *}$ & $0.0000^{* * *}$ & $0.0000^{\star * *}$ & $0.0000^{* * *}$ & $0.0000^{* * *}$ & $0.0000^{* * *}$ \\
\hline Exch (level) & 0.4521 & 0.4521 & 0.9923 & 1.0000 & 0.9772 & 0.1366 & 0.4521 & 0.4522 \\
\hline Exch ( $1^{\text {st }}$ diff $)$ & $0.0002^{\star * *}$ & $0.0002^{\star * *}$ & $0.0000^{\star * *}$ & 0.3515 & $0.0072^{\star * *}$ & $0.0001^{\star * *}$ & $0.0002^{* * *}$ & $0.0002^{* * *}$ \\
\hline LIBOR (level) & 0.1602 & 0.1602 & 0.1602 & 0.1602 & 0.1602 & 0.1602 & 0.1602 & 0.1602 \\
\hline LIBOR ( $1^{\text {st }}$ diff $)$ & $0.0000^{\star * *}$ & $0.0000^{\star * *}$ & $0.0000^{\star * *}$ & $0.0000^{* * *}$ & $0.0000^{* * *}$ & $0.0000^{\star * *}$ & $0.0000^{\star * *}$ & $0.0000^{* * *}$ \\
\hline
\end{tabular}

*** and ${ }^{* *}$ signifies significance at $1 \%$ and $5 \%$ respectively.

The results from the bounds test are reported in Table 9. The results from the F-statistic reported in column 3; indicates that only the data on Ghana is co-integrated ${ }^{* * *}$ mean a long-run relationship is established among the determinants and the money demand function of Ghana at $5 \%$ significant level). The lag structure is also recorded in the second column.

The panel test of cointegration is reported below in Table 10. The test results indicate the presence of long-run relationship among the variables of the panel.

Pedroni's cointegration tests

No. of Panel units: $16 \quad$ Regressors: 4

No. of obs.: $608 \quad$ Avg obs. per unit: 38

Data has been time-demeaned.

All test statistics are distributed $\mathrm{N}(0,1)$, under a null of no co-integration, and diverge to negative infinity (save for panel $\mathrm{v}$ ). 
Table 9. Co-integration; Bounds test and Lag order selection

\begin{tabular}{|c|c|c|c|}
\hline Country & ARDL Structure & F-statistic & Remark \\
\hline Senegal & $\left(\begin{array}{lllll}1 & 0 & 0 & 0 & 0\end{array}\right)$ & 0.477 & Not Co-integrated \\
\hline Sierra Leon & $\left(\begin{array}{lllll}2 & 1 & 0 & 0 & 0\end{array}\right)$ & 0.341 & Not Co-integrated \\
\hline Togo & $\left(\begin{array}{lllll}1 & 0 & 0 & 0 & 0\end{array}\right)$ & 0.206 & Not Co-integrated \\
\hline Nigeria & $\left(\begin{array}{lllll}1 & 0 & 0 & 0 & 0\end{array}\right)$ & 0.510 & Not Co-integrated \\
\hline Niger & $\left(\begin{array}{lllll}1 & 0 & 0 & 0 & 0\end{array}\right)$ & 0.336 & Not Co-integrated \\
\hline Mali & $\left(\begin{array}{lllll}1 & 1 & 0 & 2 & 3\end{array}\right)$ & 0.645 & Not Co-integrated \\
\hline Mauritania & $\left(\begin{array}{lllll}1 & 0 & 1 & 0 & 0\end{array}\right)$ & 0.492 & Not Co-integrated \\
\hline Liberia & $\left(\begin{array}{lllll}1 & 1 & 1 & 3 & 0\end{array}\right)$ & 4.470 & Not Co-integrated \\
\hline Ivory Coast & $\left(\begin{array}{lllll}1 & 0 & 0 & 0 & 0\end{array}\right)$ & 3.897 & Not Co-integrated \\
\hline Guinea Bissau & $\left(\begin{array}{lllll}1 & 0 & 0 & 0 & 0\end{array}\right)$ & 0.785 & Not Co-integrated \\
\hline Guinea & $\left(\begin{array}{lllll}1 & 0 & 1 & 0 & 0\end{array}\right)$ & 2.644 & Not Co-integrated \\
\hline Ghana & $\left(\begin{array}{lllll}1 & 0 & 1 & 0 & 0\end{array}\right)$ & $215.699^{* * *}$ & Co-integrated \\
\hline Gambia & $\left(\begin{array}{lllll}2 & 1 & 2 & 0 & 0\end{array}\right)$ & 2.223 & Not Co-integrated \\
\hline Cape Verde & $\left(\begin{array}{lllll}1 & 0 & 0 & 0 & 0\end{array}\right)$ & 1.659 & Not Co-integrated \\
\hline Burkina Faso & $\left(\begin{array}{lllll}1 & 0 & 0 & 0 & 0\end{array}\right)$ & 0.635 & Not Co-integrated \\
\hline Benin & $\left(\begin{array}{lllll}1 & 0 & 0 & 0 & 0\end{array}\right)$ & 0.477 & Not Co-integrated \\
\hline
\end{tabular}

Table 10. Pedroni's cointegration tests.

\begin{tabular}{ccc}
\hline Test Stats & Panel & Group \\
\hline V & 0.9291 & \\
Rho & -9.18 & -8.628 \\
T & -17.58 & -20.91 \\
Adf & -12.99 & -14.43 \\
\hline
\end{tabular}

\section{Empirical Results}

The empirical results are reported in Table 11 and Table 12 for the time series analysis for the sixteen (16) individual countries; and Table 13 reports the panel regression results for the entire sixteen (16) countries; the sub-samples of French speaking countries and others (English and Portuguese speakers). The results reported in both Table 11 and Table 12 indicates short run dynamics except for Ghana since the ARDL bounds test proved not co-integrated. From Table 11 and Table 12; an increase real income (real GDP) has significant and positive effect on money demand in all the sixteen (16) countries in the short run. Similarly; inflation is recorded to have positive and significant effect on money demand in Nigeria and Mauritania in the short run. Exchange rate also has significant negative impact on money demand in Gambia, Sierra Leon, Mauritania and Liberia. The foreign interest rate, LIBOR has a negative and significant effect on money demand only in Ivory Coast in the short run. In the remaining fifteen (15) 
M. Asiedu et al.

Table 11. Regression results.

\begin{tabular}{|c|c|c|c|c|c|c|c|c|}
\hline & $\begin{array}{l}\text { Senegal } \\
\text { LnRM2 }\end{array}$ & $\begin{array}{l}\text { Sierra L } \\
\text { lnRM2 }\end{array}$ & $\begin{array}{c}\text { Togo } \\
\text { lnRM2 }\end{array}$ & $\begin{array}{l}\text { Nigeria } \\
\text { lnRM2 }\end{array}$ & $\begin{array}{l}\text { Niger } \\
\text { lnRM2 }\end{array}$ & $\begin{array}{c}\text { Mali } \\
\text { lnRM2 }\end{array}$ & $\begin{array}{c}\text { Mauritania } \\
\text { lnRM2 }\end{array}$ & $\begin{array}{l}\text { Liberia } \\
\text { lnRM2 }\end{array}$ \\
\hline \multirow[t]{2}{*}{ L.lnRM2 } & -0.002 & 0.026 & 0.004 & -0.000 & 0.001 & -0.002 & $0.318^{*}$ & 0.003 \\
\hline & $(0.001)$ & $(0.094)$ & $(0.004)$ & $(0.001)$ & $(0.003)$ & $(0.002)$ & $(0.163)$ & $(0.004)$ \\
\hline \multirow[t]{2}{*}{$\mathrm{L} 2 . \ln \mathrm{RM} 2$} & & $0.224^{* *}$ & & & & & & \\
\hline & & $(0.096)$ & & & & & & \\
\hline \multirow[t]{2}{*}{ Infl } & 0.003 & -0.001 & 0.006 & $0.002^{* * *}$ & 0.009 & -0.011 & $0.006^{* *}$ & -0.036 \\
\hline & $(0.004)$ & $(0.007)$ & $(0.053)$ & $(0.000)$ & $(0.034)$ & $(0.011)$ & $(0.003)$ & $(0.091)$ \\
\hline \multirow[t]{2}{*}{ LnRGDP } & $0.954^{* * *}$ & $0.453^{* * *}$ & $0.951^{* * *}$ & $0.957^{* * *}$ & $0.910^{* * *}$ & $0.920^{* * *}$ & $0.935^{* * *}$ & $0.544^{* * *}$ \\
\hline & $(0.001)$ & $(0.048)$ & $(0.003)$ & $(0.001)$ & $(0.002)$ & $(0.002)$ & $(0.002)$ & $(0.002)$ \\
\hline \multirow[t]{2}{*}{ Exch } & -0.000 & $-0.000^{* *}$ & 0.002 & $0.000^{* * *}$ & 0.000 & -0.001 & $-0.042^{*}$ & $-0.047^{*}$ \\
\hline & $(0.000)$ & $(0.000)$ & $(0.003)$ & $(0.000)$ & $(0.002)$ & $(0.001)$ & $(0.020)$ & $(0.025)$ \\
\hline \multirow[t]{2}{*}{ LIBOR } & 0.004 & -0.155 & 0.065 & -0.004 & 0.071 & -0.036 & -0.002 & -0.397 \\
\hline & $(0.015)$ & $(0.102)$ & $(0.131)$ & $(0.003)$ & $(0.101)$ & $(0.076)$ & $(0.025)$ & $(0.271)$ \\
\hline \multirow[t]{2}{*}{ L.Infl } & & & & & & $-0.034^{\star \star \star}$ & 0.002 & \\
\hline & & & & & & $(0.011)$ & $(0.003)$ & \\
\hline \multirow[t]{2}{*}{ L2.Infl } & & & & & & & $0.006^{*}$ & \\
\hline & & & & & & & $(0.003)$ & \\
\hline \multirow[t]{2}{*}{ L3.Infl } & & & & & & & $-0.006^{\star}$ & \\
\hline & & & & & & & $(0.003)$ & \\
\hline \multirow[t]{2}{*}{ L.Exch } & & & & & & $0.005^{* *}$ & 0.052 & \\
\hline & & & & & & $(0.002)$ & $(0.033)$ & \\
\hline \multirow[t]{2}{*}{ L2.Exch } & & & & & & $-0.005^{\star * *}$ & 0.008 & \\
\hline & & & & & & $(0.001)$ & $(0.032)$ & \\
\hline \multirow[t]{2}{*}{ L3.Exch } & & & & & & & 0.049 & \\
\hline & & & & & & & $(0.032)$ & \\
\hline \multirow[t]{2}{*}{ L4.Exch } & & & & & & & $-0.089^{\star * *}$ & \\
\hline & & & & & & & $(0.024)$ & \\
\hline \multirow[t]{2}{*}{ L.LIBOR } & & & & & & -0.070 & 0.017 & \\
\hline & & & & & & $(0.132)$ & $(0.036)$ & \\
\hline \multirow[t]{2}{*}{ L2.LIBOR } & & & & & & 0.103 & -0.034 & \\
\hline & & & & & & $(0.131)$ & $(0.037)$ & \\
\hline \multirow[t]{2}{*}{ L3.LIBOR } & & & & & & $-0.176^{\star *}$ & $-0.062^{\star *}$ & \\
\hline & & & & & & $(0.073)$ & $(0.027)$ & \\
\hline \multirow[t]{2}{*}{ L.InRGDP } & & & & & & & $-0.303^{\star}$ & \\
\hline & & & & & & & $(0.153)$ & \\
\hline \multirow[t]{2}{*}{ _cons } & 0.084 & 0.910 & -1.522 & $-0.105^{\star \star \star}$ & -0.800 & $2.038^{\star * *}$ & $0.695^{\star *}$ & $5.877^{\star *}$ \\
\hline & $(0.216)$ & $(0.706)$ & $(1.855)$ & $(0.026)$ & (1.408) & $(0.452)$ & $(0.229)$ & $(2.476)$ \\
\hline Obs. & 37 & 36 & 37 & 37 & 37 & 35 & 29 & 35 \\
\hline
\end{tabular}




\section{Continued}

\begin{tabular}{|c|c|c|c|c|c|c|c|c|}
\hline R-squared & 0.985 & 0.808 & 0.984 & 0.957 & 0.986 & 0.985 & 0.978 & 0.978 \\
\hline BG LM test & 0.5163 & 0.8024 & 0.3386 & 0.3046 & 0.8085 & 0.0535 & & 0.1148 \\
\hline B-Pagan Hettest & 0.7483 & 0.0132 & 0.0000 & 0.1259 & 0.0014 & 0.3727 & & 0.8195 \\
\hline Normality test & 0.7483 & 0.3491 & 0.0675 & 0.9476 & 0.1259 & 0.6243 & & 0.9158 \\
\hline CUSUM & Stable & Stable & Stable & Stable & Stable & Stable & Stable & Stable \\
\hline CUSUMsq & Not stable & Stable & Not stable & Not stable & Not stable & Not stable & Stable & Stable \\
\hline
\end{tabular}

Table 12. Regression results.

\begin{tabular}{|c|c|c|c|c|c|c|c|c|}
\hline & $\begin{array}{c}\text { Guinea B } \\
\ln R M 2\end{array}$ & $\begin{array}{l}\text { Guinea } \\
\ln R M 2\end{array}$ & $\begin{array}{l}\text { Cape V } \\
\text { lnRM2 }\end{array}$ & $\begin{array}{l}\text { Gambia } \\
\ln R M 2\end{array}$ & $\begin{array}{c}\text { BurkinaF. } \\
\ln R M 2\end{array}$ & $\begin{array}{l}\text { Benin } \\
\ln R M 2\end{array}$ & $\begin{array}{l}\text { Ghana } \\
\text { lnRM2 }\end{array}$ & $\begin{array}{c}\text { Ivory C. } \\
\ln R M 2\end{array}$ \\
\hline \multirow[t]{2}{*}{ L.lnRM2 } & 0.001 & $0.487^{\star * *}$ & -0.002 & 0.127 & 0.002 & -0.002 & $-1.002^{\star * *}$ & 0.000 \\
\hline & $(0.002)$ & $(0.172)$ & $(0.002)$ & $(0.155)$ & $(0.002)$ & $(0.001)$ & $(0.001)$ & $(0.000)$ \\
\hline \multirow[t]{2}{*}{ L2.lnRM2 } & & & & $0.383^{* *}$ & & & & \\
\hline & & & & $(0.149)$ & & & & \\
\hline \multirow[t]{2}{*}{ Infl } & -0.001 & -0.000 & -0.004 & -0.000 & 0.012 & 0.003 & & -0.001 \\
\hline & $(0.002)$ & $(0.001)$ & $(0.007)$ & $(0.000)$ & $(0.022)$ & $(0.004)$ & & $(0.003)$ \\
\hline \multirow[t]{2}{*}{ LnRGDP } & $0.980^{* * *}$ & $0.930^{* * *}$ & $0.998^{* * *}$ & $0.931^{\star * *}$ & $0.934^{* * *}$ & $0.954^{* * *}$ & & $0.956^{* * *}$ \\
\hline & $(0.002)$ & $(0.002)$ & $(0.002)$ & $(0.003)$ & $(0.002)$ & $(0.001)$ & & $(0.000)$ \\
\hline \multirow[t]{2}{*}{ Exch } & -0.000 & 0.000 & 0.009 & $0.004^{*}$ & 0.001 & -0.000 & & -0.000 \\
\hline & $(0.000)$ & $(0.000)$ & $(0.006)$ & $(0.002)$ & $(0.001)$ & $(0.000)$ & & $(0.000)$ \\
\hline \multirow[t]{3}{*}{ LIBOR } & -0.032 & 0.002 & 0.009 & 0.008 & -0.013 & 0.004 & & $-0.019^{*}$ \\
\hline & $(0.023)$ & $(0.013)$ & $(0.027)$ & $(0.007)$ & $(0.037)$ & $(0.015)$ & & $(0.011)$ \\
\hline & & & & $(0.138)$ & & & & \\
\hline \multirow[t]{2}{*}{ L.Infl } & & & & $-0.002^{* * *}$ & & & & \\
\hline & & & & $(0.000)$ & & & & \\
\hline \multirow[t]{2}{*}{ _cons } & 0.324 & -0.029 & -0.795 & -0.057 & -0.771 & 0.084 & & \\
\hline & $(0.280)$ & $(0.105)$ & $(0.601)$ & $(0.071)$ & $(0.530)$ & $(0.216)$ & & \\
\hline \multirow[t]{2}{*}{ LR:Infl } & & & & & & & 0.003 & \\
\hline & & & & & & & $(0.004)$ & \\
\hline \multirow[t]{2}{*}{ LR:InRGDP } & & & & & & & $0.952^{* * *}$ & \\
\hline & & & & & & & $(0.002)$ & \\
\hline \multirow[t]{2}{*}{ LR:Exch } & & & & & & & -0.000 & \\
\hline & & & & & & & $(0.000)$ & \\
\hline \multirow[t]{2}{*}{ LR: LIBOR } & & & & & & & 0.004 & \\
\hline & & & & & & & $(0.015)$ & \\
\hline \multirow[t]{2}{*}{ SR:_cons } & & & & & & & 0.084 & 0.239 \\
\hline & & & & & & & $(0.216)$ & $(0.179)$ \\
\hline Obs. & 37 & 37 & 37 & 36 & 37 & 37 & 37 & 37 \\
\hline R-squared & 0.989 & 0.982 & 0.995 & 0.984 & 0.967 & 0.983 & 0.987 & 0.984 \\
\hline BG LM test & 0.5104 & 0.0067 & 0.4563 & 0.2657 & 0.1431 & 0.5163 & 0.5163 & 0.5515 \\
\hline B-P Hettest & 0.1640 & 0.0000 & 0.0000 & 0.0639 & 0.0030 & 0.7483 & 0.7483 & 0.0313 \\
\hline Normality & 0.4163 & 0.0000 & 0.0000 & 0.0041 & 0.0219 & 0.8488 & 0.8488 & 0.4229 \\
\hline cusum & Stable & Stable & Stable & Stable & Stable & Stable & Not Stable & Stable \\
\hline cusumsq & Not stable & Stable & Not stable & Stable & Stable & Not stable & Stable & Not stable \\
\hline
\end{tabular}

Standard errors are in parenthesis; ${ }^{* *} p<0.01,{ }^{* *} p<0.05,{ }^{*} p<0.1$. 
countries, it is insignificant. The results from the long-run regression for Ghana indicates that only real income (real GDP) positively and significantly affects money demand.

Based on the results from the panel co-integration results reported in Table 10; the results for all the sixteen (16) countries and the sub-samples of French speaking countries and other (made up English and Portuguese speaking countries) are reported in Table 11 and Table 12. The result from Table 13 indicates that in the long-run real income has positive and significant effect on money demand. This is also true among the sub-samples of French speaking countries and others. The foreign interest rate, LIBOR is also found to negatively and significantly affect money demand among all the sixteen countries.

Table 13. Regression results.

\begin{tabular}{|c|c|c|c|c|}
\hline & $\begin{array}{l}\text { All countries } \\
\text { D.lnRM2 }\end{array}$ & $\begin{array}{l}\text { Full sample } \\
\text { D.lnRM2 }\end{array}$ & $\begin{array}{l}\text { Others } \\
\text { D.lnRM2 }\end{array}$ & $\begin{array}{c}\text { French } \\
\text { D.lnRM2 }\end{array}$ \\
\hline \multirow[t]{2}{*}{ Infl } & 0.000 & 0.000 & 0.000 & 0.001 \\
\hline & $(0.000)$ & $(0.000)$ & $(0.000)$ & $(0.005)$ \\
\hline \multirow[t]{2}{*}{ LnRGDP } & $0.949^{* * *}$ & $0.949^{* * *}$ & $0.979^{* * *}$ & $0.950^{\star * *}$ \\
\hline & $(0.002)$ & $(0.002)$ & $(0.002)$ & $(0.002)$ \\
\hline \multirow[t]{2}{*}{ Exch } & 0.000 & 0.000 & -0.000 & -0.000 \\
\hline & $(0.000)$ & $(0.000)$ & $(0.000)$ & $(0.000)$ \\
\hline \multirow[t]{2}{*}{ LIBOR } & $-0.005^{\star * *}$ & $-0.005^{\star * *}$ & -0.002 & 0.000 \\
\hline & $(0.001)$ & $(0.001)$ & $(0.003)$ & $(0.010)$ \\
\hline \multirow[t]{2}{*}{ Senegal:_ec } & $-0.151^{*}$ & & & \\
\hline & $(0.084)$ & & & \\
\hline \multirow[t]{2}{*}{ D.Infl } & 0.026 & & & \\
\hline & $(0.091)$ & & & \\
\hline \multirow[t]{2}{*}{ D.lnRGDP } & $0.775^{\star * *}$ & & & \\
\hline & $(0.078)$ & & & \\
\hline \multirow[t]{2}{*}{ D.Exch } & -0.002 & & & \\
\hline & $(0.012)$ & & & \\
\hline \multirow[t]{2}{*}{ D.LIBOR } & -0.334 & & & \\
\hline & $(0.471)$ & & & \\
\hline \multirow[t]{2}{*}{ Cons } & 0.249 & & & \\
\hline & $(0.551)$ & & & \\
\hline \multirow[t]{2}{*}{ Sierra L:_ec } & $-0.714^{\star * *}$ & & & \\
\hline & $(0.129)$ & & & \\
\hline \multirow[t]{2}{*}{ D.Infl } & -0.000 & & & \\
\hline & $(0.000)$ & & & \\
\hline D. $\ln R G D P$ & $0.270^{\star \star}$ & & & \\
\hline
\end{tabular}




\section{Continued}

\begin{tabular}{|c|c|}
\hline & $(0.122)$ \\
\hline D.Exch & $\begin{array}{c}0.000 \\
(0.000)\end{array}$ \\
\hline D.LIBOR & $\begin{array}{l}-0.010 \\
(0.007)\end{array}$ \\
\hline Cons & $\begin{array}{c}-0.031^{\star} \\
(0.016)\end{array}$ \\
\hline Togo:_ec & $\begin{array}{c}-1.114^{* * *} \\
(0.167)\end{array}$ \\
\hline D.Infl & $\begin{array}{c}0.006 \\
(0.038)\end{array}$ \\
\hline D.lnRGDP & $\begin{array}{l}-0.108 \\
(0.159)\end{array}$ \\
\hline D.Exch & $\begin{array}{c}0.001 \\
(0.006)\end{array}$ \\
\hline D.LIBOR & $\begin{array}{c}0.023 \\
(0.280)\end{array}$ \\
\hline Cons & $\begin{array}{l}-0.161 \\
(0.331)\end{array}$ \\
\hline Nigeria:_ec & $\begin{array}{c}-0.310^{* * *} \\
(0.098)\end{array}$ \\
\hline D.Infl & $\begin{array}{c}0.002^{* * *} \\
(0.000)\end{array}$ \\
\hline D.lnRGDP & $\begin{array}{l}0.661^{\star * *} \\
(0.094)\end{array}$ \\
\hline D.Exch & $\begin{array}{l}0.001^{*} \\
(0.000)\end{array}$ \\
\hline D.LIBOR & $\begin{array}{l}-0.003 \\
(0.005)\end{array}$ \\
\hline Cons & $\begin{array}{l}-0.002 \\
(0.007)\end{array}$ \\
\hline Niger:_ec & $\begin{array}{c}-0.217^{\star *} \\
(0.102)\end{array}$ \\
\hline :D.Infl & $\begin{array}{l}-0.004 \\
(0.026)\end{array}$ \\
\hline D.lnRGDP & $\begin{array}{l}0.706^{\star * *} \\
(0.095)\end{array}$ \\
\hline
\end{tabular}




\section{Continued}

\begin{tabular}{|c|c|}
\hline D.Exch & $\begin{array}{l}0.009^{*} \\
(0.005)\end{array}$ \\
\hline D.LIBOR & $\begin{array}{l}-0.196 \\
(0.281)\end{array}$ \\
\hline Cons & $\begin{array}{l}-0.247 \\
(0.329)\end{array}$ \\
\hline Mali:_ec & $\begin{array}{c}-0.233^{* *} \\
(0.098)\end{array}$ \\
\hline D.Infl & $\begin{array}{c}0.010 \\
(0.008)\end{array}$ \\
\hline D.lnRGDP & $\begin{array}{c}0.705^{\star * *} \\
(0.091)\end{array}$ \\
\hline D.Exch & $\begin{array}{l}-0.002 \\
(0.001)\end{array}$ \\
\hline D.LIBOR & $\begin{array}{l}-0.072 \\
(0.066)\end{array}$ \\
\hline Cons & $\begin{array}{c}0.082 \\
(0.082)\end{array}$ \\
\hline Mauritania:_ec & $\begin{array}{c}-0.513^{* * *} \\
(0.143)\end{array}$ \\
\hline D.Infl & $\begin{array}{c}0.001 \\
(0.002)\end{array}$ \\
\hline D.lnRGDP & $\begin{array}{c}0.457^{* * *} \\
(0.135)\end{array}$ \\
\hline D.Exch & $\begin{array}{c}0.007 \\
(0.016)\end{array}$ \\
\hline D.LIBOR & $\begin{array}{c}0.004 \\
(0.016)\end{array}$ \\
\hline Cons & $\begin{array}{c}0.002 \\
(0.024)\end{array}$ \\
\hline Liberia:_ec & $\begin{array}{l}-0.122 \\
(0.083)\end{array}$ \\
\hline D.Infl & $\begin{array}{c}0.045^{* * *} \\
(0.014)\end{array}$ \\
\hline D.lnRGDP & $\begin{array}{c}0.845^{\star * *} \\
(0.080)\end{array}$ \\
\hline D.Exch & $\begin{array}{c}0.004 \\
(0.004)\end{array}$ \\
\hline D.LIBOR & 0.053 \\
\hline
\end{tabular}




\section{Continued}

\begin{tabular}{|c|c|}
\hline & $(0.032)$ \\
\hline \multirow[t]{2}{*}{ Cons } & -0.031 \\
\hline & $(0.069)$ \\
\hline \multirow[t]{2}{*}{ Ivory Coast:_ec } & -0.012 \\
\hline & $(0.030)$ \\
\hline \multirow[t]{2}{*}{ D.Infl } & -0.000 \\
\hline & $(0.003)$ \\
\hline \multirow[t]{2}{*}{ D.lnRGDP } & $0.944^{* * *}$ \\
\hline & $(0.029)$ \\
\hline \multirow[t]{2}{*}{ D.Exch } & -0.000 \\
\hline & $(0.001)$ \\
\hline \multirow[t]{2}{*}{ D.LIBOR } & 0.020 \\
\hline & $(0.028)$ \\
\hline \multirow[t]{2}{*}{ Cons } & 0.014 \\
\hline & $(0.032)$ \\
\hline \multirow{2}{*}{ Guinea B:_ec } & -0.087 \\
\hline & $(0.088)$ \\
\hline \multirow[t]{2}{*}{ D.Infl } & 0.000 \\
\hline & $(0.002)$ \\
\hline \multirow[t]{2}{*}{ D.lnRGDP } & $0.897^{\star * *}$ \\
\hline & $(0.085)$ \\
\hline \multirow[t]{2}{*}{ D.Exch } & -0.000 \\
\hline & $(0.001)$ \\
\hline \multirow[t]{2}{*}{ D.LIBOR } & -0.036 \\
\hline & $(0.055)$ \\
\hline \multirow[t]{2}{*}{ Cons } & -0.018 \\
\hline & $(0.068)$ \\
\hline \multirow[t]{2}{*}{ Guinea:__ec } & -0.104 \\
\hline & $(0.078)$ \\
\hline \multirow[t]{2}{*}{ D.Infl } & 0.001 \\
\hline & $(0.001)$ \\
\hline \multirow[t]{2}{*}{ D.lnRGDP } & $0.833^{\star * *}$ \\
\hline & $(0.074)$ \\
\hline \multirow[t]{2}{*}{ D.Exch } & -0.000 \\
\hline & $(0.000)$ \\
\hline \multirow[t]{2}{*}{ D.LIBOR } & 0.005 \\
\hline & $(0.017)$ \\
\hline \multirow[t]{2}{*}{ Cons } & 0.007 \\
\hline & $(0.028)$ \\
\hline
\end{tabular}




\section{Continued}

\begin{tabular}{|c|c|}
\hline Ghana:_ec & $\begin{array}{c}-0.453^{* * *} \\
(0.125)\end{array}$ \\
\hline D.Infl & $\begin{array}{c}0.000 \\
(0.000)\end{array}$ \\
\hline D.lnRGDP & $\begin{array}{c}0.517^{\star * *} \\
(0.118)\end{array}$ \\
\hline D.Exch & $\begin{array}{c}0.005 \\
(0.007)\end{array}$ \\
\hline D.LIBOR & $\begin{array}{c}0.001 \\
(0.001)\end{array}$ \\
\hline Cons & $\begin{array}{c}0.001 \\
(0.004)\end{array}$ \\
\hline Gambia:_ec & $\begin{array}{c}-0.438^{* * *} \\
(0.121)\end{array}$ \\
\hline D.Infl & $\begin{array}{l}0.001^{*} \\
(0.000)\end{array}$ \\
\hline D.lnRGDP & $\begin{array}{c}0.517^{* * *} \\
(0.114)\end{array}$ \\
\hline D.Exch & $\begin{array}{c}0.005 \\
(0.006)\end{array}$ \\
\hline D.LIBOR & $\begin{array}{l}0.021^{*} \\
(0.011)\end{array}$ \\
\hline Cons & $\begin{array}{l}-0.009 \\
(0.016)\end{array}$ \\
\hline Cape V:_ec & $\begin{array}{l}-0.083 \\
(0.065)\end{array}$ \\
\hline D.Infl & $\begin{array}{l}-0.004 \\
(0.005)\end{array}$ \\
\hline D.lnRGDP & $\begin{array}{c}0.918^{\star * *} \\
(0.063)\end{array}$ \\
\hline D.Exch & $\begin{array}{c}0.003 \\
(0.012)\end{array}$ \\
\hline D.LIBOR & $\begin{array}{l}-0.072 \\
(0.076)\end{array}$ \\
\hline Cons & $\begin{array}{l}-0.033 \\
(0.092)\end{array}$ \\
\hline Burkina F:_ec & $\begin{array}{c}-0.547^{\star * *} \\
(0.151)\end{array}$ \\
\hline D.Infl & -0.018 \\
\hline
\end{tabular}




\section{Continued}

\begin{tabular}{|c|c|c|c|c|}
\hline & $(0.020)$ & & & \\
\hline \multirow[t]{2}{*}{ D.lnRGDP } & $0.418^{\star * *}$ & & & \\
\hline & $(0.141)$ & & & \\
\hline \multirow[t]{2}{*}{ D.Exch } & 0.001 & & & \\
\hline & $(0.002)$ & & & \\
\hline \multirow[t]{2}{*}{ D.LIBOR } & -0.004 & & & \\
\hline & $(0.102)$ & & & \\
\hline \multirow[t]{2}{*}{ Cons } & -0.213 & & & \\
\hline & (0.129) & & & \\
\hline \multirow[t]{2}{*}{ Benin:_ec } & $-0.947^{* * *}$ & & & \\
\hline & $(0.168)$ & & & \\
\hline \multirow[t]{2}{*}{ D.Infl } & 0.000 & & & \\
\hline & $(0.003)$ & & & \\
\hline \multirow[t]{2}{*}{ D.lnRGDP } & 0.055 & & & \\
\hline & $(0.160)$ & & & \\
\hline \multirow[t]{2}{*}{ D.Exch } & 0.000 & & & \\
\hline & $(0.001)$ & & & \\
\hline \multirow[t]{2}{*}{ D.LIBOR } & -0.029 & & & \\
\hline & $(0.035)$ & & & \\
\hline \multirow[t]{2}{*}{ Cons } & -0.040 & & & \\
\hline & $(0.042)$ & & & \\
\hline \multirow[t]{2}{*}{ SR:_ec } & & $-0.378^{* * *}$ & $-0.326^{\star *}$ & $-0.445^{* * *}$ \\
\hline & & $(0.081)$ & $(0.128)$ & $(0.157)$ \\
\hline \multirow[t]{2}{*}{ SR:D.Infl } & & 0.004 & 0.006 & -0.001 \\
\hline & & $(0.004)$ & $(0.006)$ & $(0.003)$ \\
\hline \multirow[t]{2}{*}{ SR:D.lnRGDP } & & $0.588^{\star * *}$ & $0.642^{\star * *}$ & $0.516^{* * *}$ \\
\hline & & $(0.078)$ & $(0.122)$ & $(0.147)$ \\
\hline \multirow[t]{2}{*}{ SR:D.Exch } & & $0.002^{* *}$ & $0.002^{* *}$ & 0.002 \\
\hline & & $(0.001)$ & $(0.001)$ & $(0.001)$ \\
\hline \multirow[t]{2}{*}{ SR:D.LIBOR } & & -0.039 & 0.005 & -0.030 \\
\hline & & $(0.024)$ & $(0.014)$ & $(0.025)$ \\
\hline \multirow[t]{2}{*}{ SR:_cons } & & -0.027 & $-0.024^{* * *}$ & -0.009 \\
\hline & & $(0.028)$ & $(0.008)$ & $(0.039)$ \\
\hline Obs. & 592 & 592 & 259 & 296 \\
\hline
\end{tabular}

\section{Test for Stability}

Following Asongu et al. (2019a) an economy is said to be experiencing money demand stability if the results from both the CUSUM test and CUSUM squared test are stable at 5\% significance level. Partial stability exists when only one of 
the tests (CUSUM test or CUSUM squared test) is stable. The results from the CUSUM test and CUSUM squared test show significant divergence in the stability of money demand among the sixteen (16) countries in West Africa. From both the CUSUM and CUSUM squared test graphs, only five (5) countries; namely: Gambia, Burkina Faso, Sierra Leon, Mauritania and Liberia have stability in their money demand functions whilst the remaining eleven (11) exhibited partial stability. These results are consistent with the findings of Asongu et al. (2019a) who also found significant divergence in the stability of money demand function among Southern Africa development countries. The evidence from these test of stability especially among the remaining eleven (11) countries points to the fact that despite the conscious efforts of most countries in west Africa through policies and programs such as the Economic Recovery Program (ERP), Structural Adjustment Program (SAP) and all the policies towards financial liberalization and financial deepening; eleven (11) of these are still experiencing significant instability. This means that in the event of an adoption of a single currency through a monetary union as being proposed by the West African Monetary Zone, special or specific considerations must be made for these eleven (11) countries when designing common monetary policies and tools for the monetary zone.

The CUSUM test and CUSUM squared test for stability graphs for the sixteen (16) countries are presented below.

Figure 1(a) and Figure 1(b) below reports the stability results from the CUSUM test and CUSUM squared test respectively for Senegal. Partial stability is recorded in the money demand function based on the CUSUM graph while the CUSUM squared test rejects the presence of stability in the money demand function.

Figure 2(a) and Figure 2(b) below reports the stability results from the CUSUM test and CUSUM squared test respectively for Sierra Leon. Total stability is recorded in the money demand function based on the results from both the CUSUM squared test graph and the CUSUM graph. Both graphs are significant at $5 \%$.
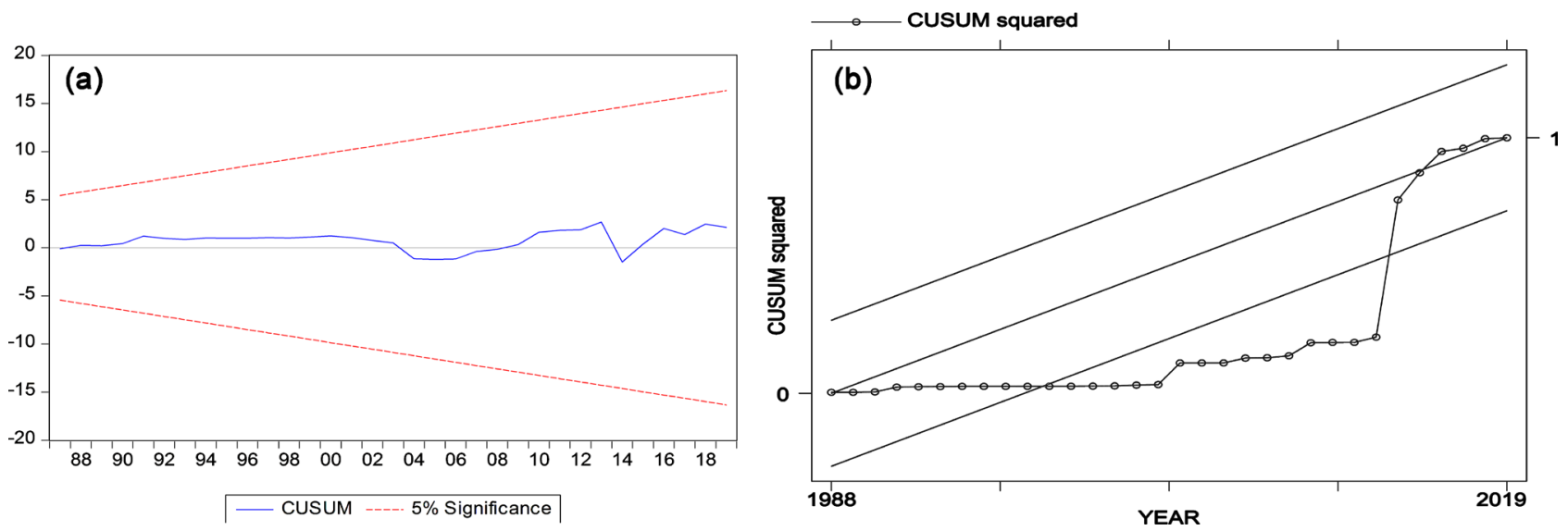

Figure 1. Stability graph of SENEGAL. 
Figure 3(a) and Figure 3(b) below reports the stability results from the CUSUM test and CUSUM squared test respectively for Togo. Partial stability is recorded in the money demand function based on the CUSUM graph while the CUSUM squared test rejects the presence of stability in the money demand function.

Figure 4(a) and Figure 4(b) below reports the stability results from the CUSUM test and CUSUM squared test respectively for Nigeria. Partial stability is recorded in the money demand function based on the CUSUM graph while the CUSUM squared test rejects the presence of stability in the money demand function.

Figure 5(a) and Figure 5(b) below reports the stability results from the CUSUM test and CUSUM squared test respectively for Nigeria. Partial stability is recorded in the money demand function based on the CUSUM graph while the CUSUM squared test rejects the presence of stability in the money demand function.

Figure 6(a) and Figure 6(b) below reports the stability results from the CUSUM test and CUSUM squared test respectively for Mali. Partial stability is
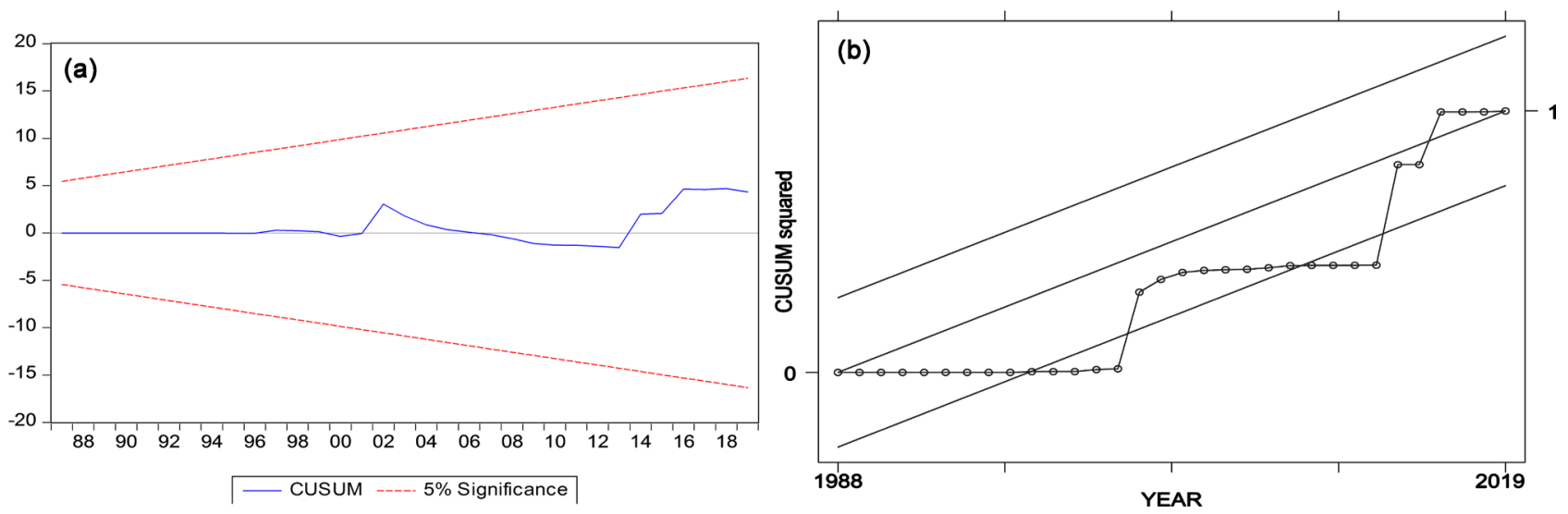

Figure 2. Stability graph of Sierra Leon.
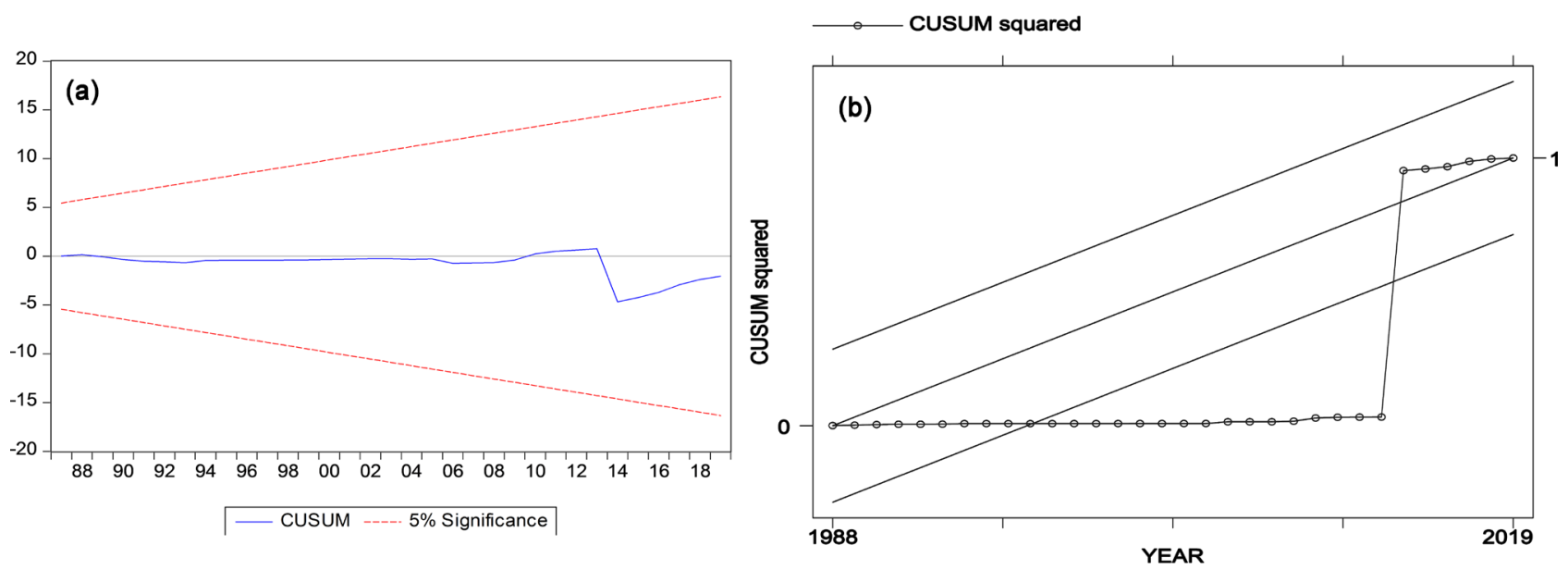

Figure 3. Stability graph of Togo. 

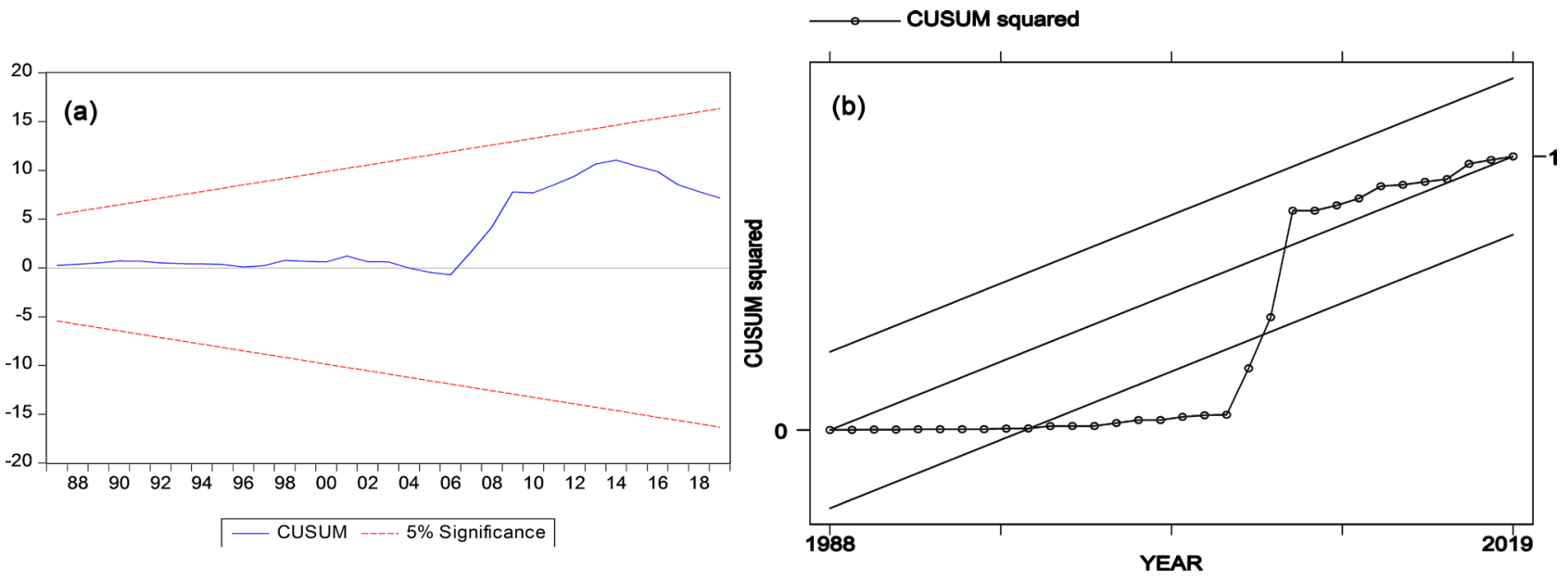

Figure 4. Stability graph of Nigeria.
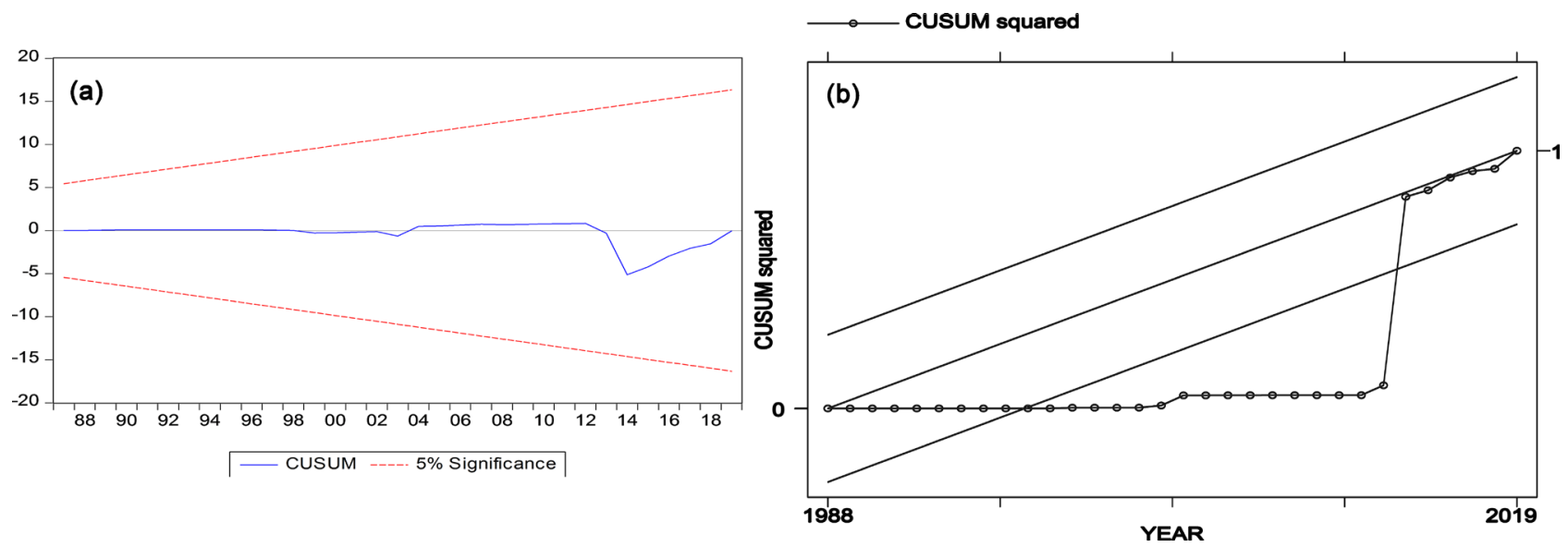

Figure 5. Stability graph of Niger.
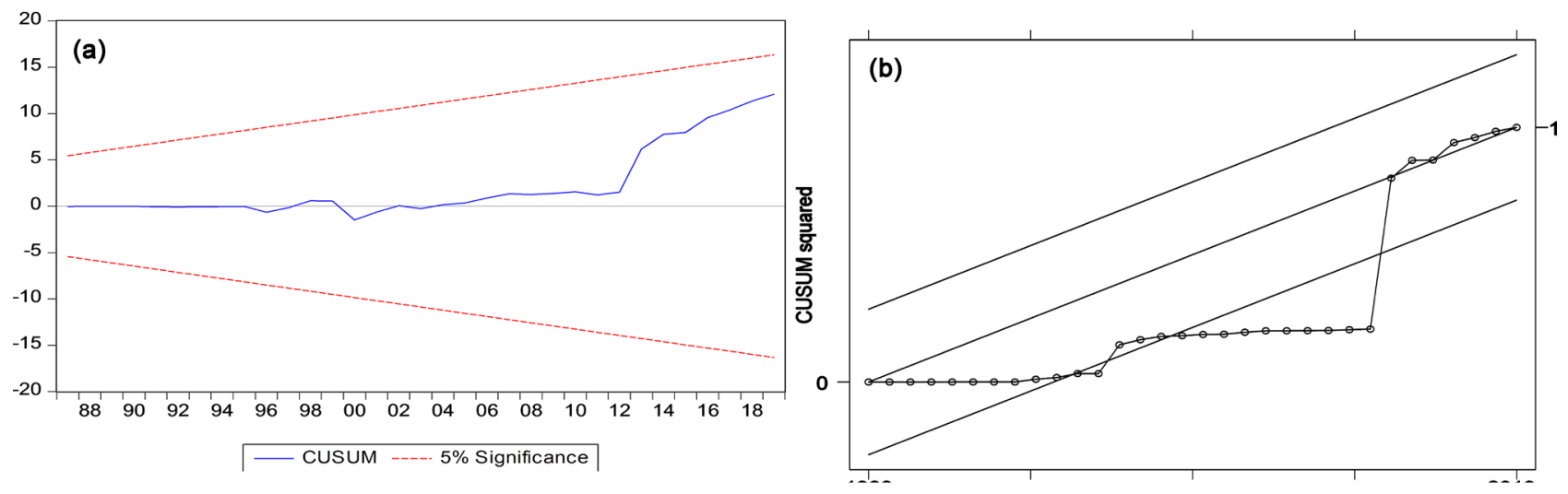

Figure 6. Stability graph of Mali.

recorded in the money demand function based on the CUSUM graph while the CUSUM squared test rejects the presence of stability in the money demand function.

Figure $7(\mathrm{a})$ and Figure $7(\mathrm{~b})$ below reports the stability results from the 
CUSUM test and CUSUM squared test respectively for Mauritania. Total stability is recorded in the money demand function based on the results from both the CUSUM squared test graph and the CUSUM graph. Both graphs are significant at $5 \%$.

Figure 8(a) and Figure 8(b) below reports the stability results from the CUSUM test and CUSUM squared test respectively for Liberia. Total stability is recorded in the money demand function based on the results from both the CUSUM squared test graph and the CUSUM graph. Both graphs are significant at $5 \%$.

Figure 9(a) and Figure 9(b) below reports the stability results from the CUSUM test and CUSUM squared test respectively for Ivory Coast. Partial stability is recorded in the money demand function based on the CUSUM graph while the CUSUM squared test rejects the presence of stability in the money demand function.

Figure 10(a) and Figure 10(b) below reports the stability results from the CUSUM test and CUSUM squared test respectively for Guinea Bissau. Partial stability is recorded in the money demand function based on the CUSUM graph
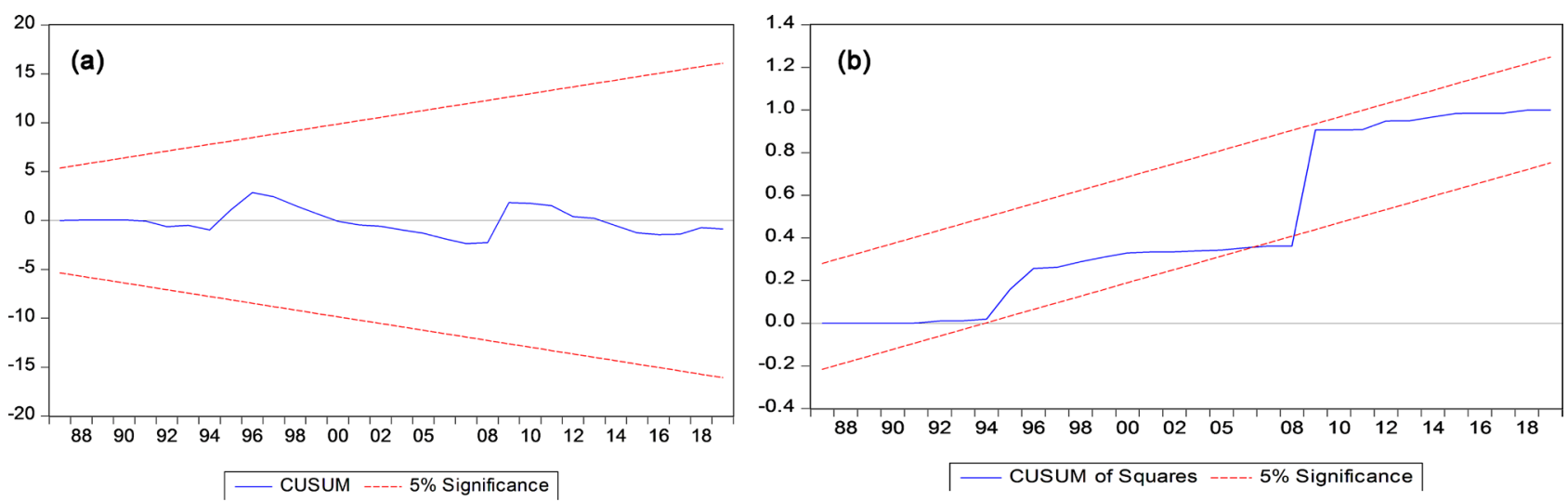

Figure 7. Stability graph of Mauritania.
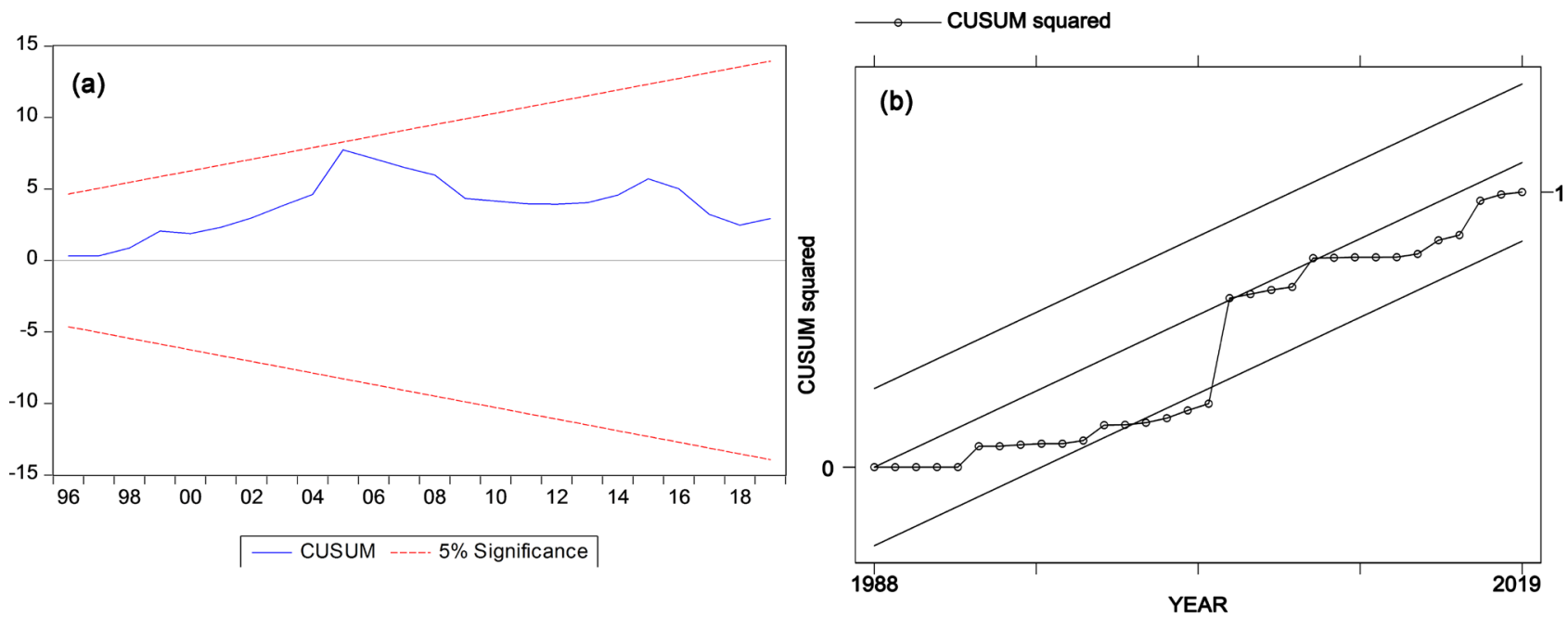

Figure 8. Stability graph of Liberia. 


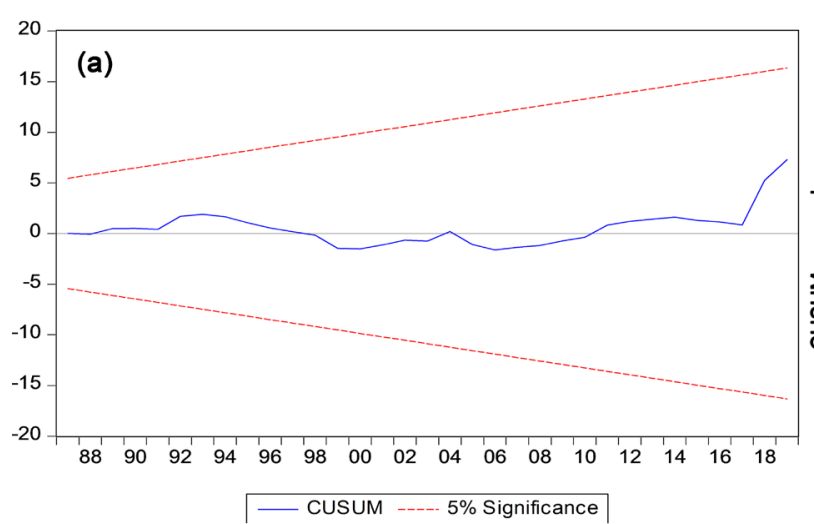

Figure 9. Stability graph of Ivory Coast.

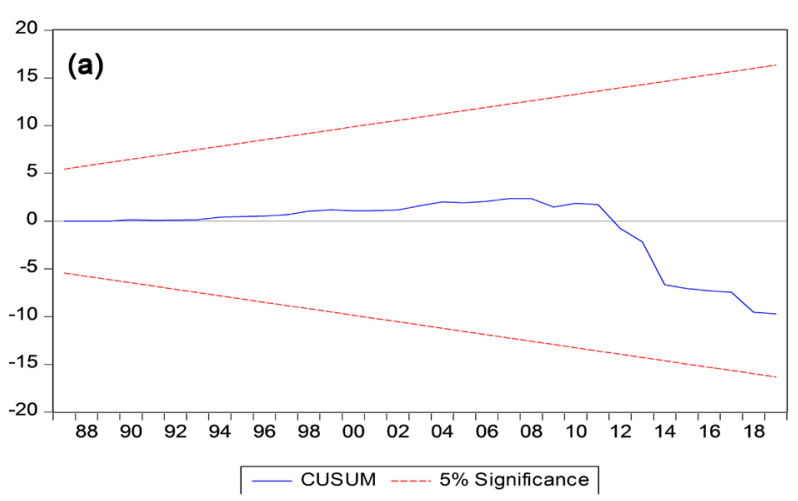

Figure 10. Stability graph of Guinea Bissau.

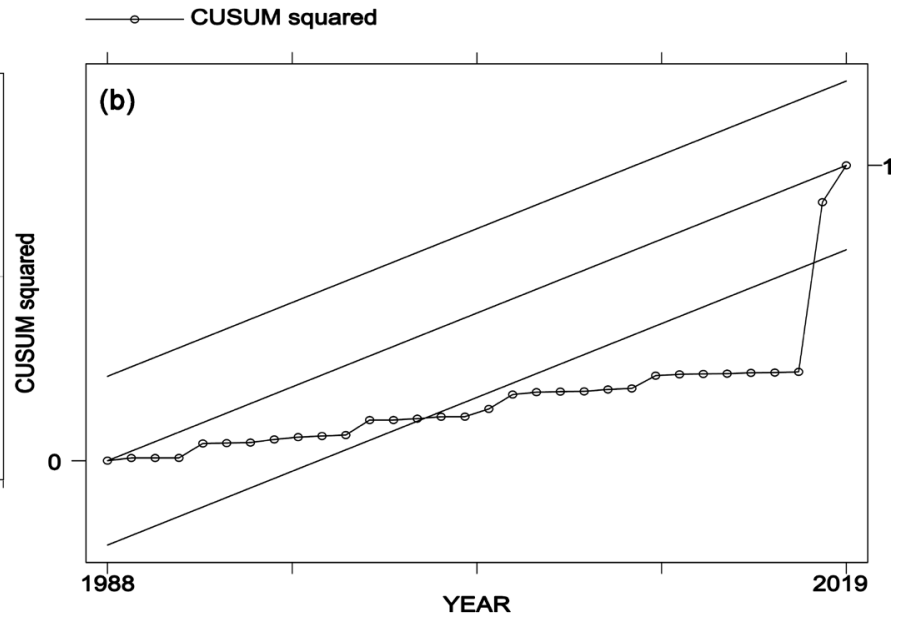

- CUSUM squared

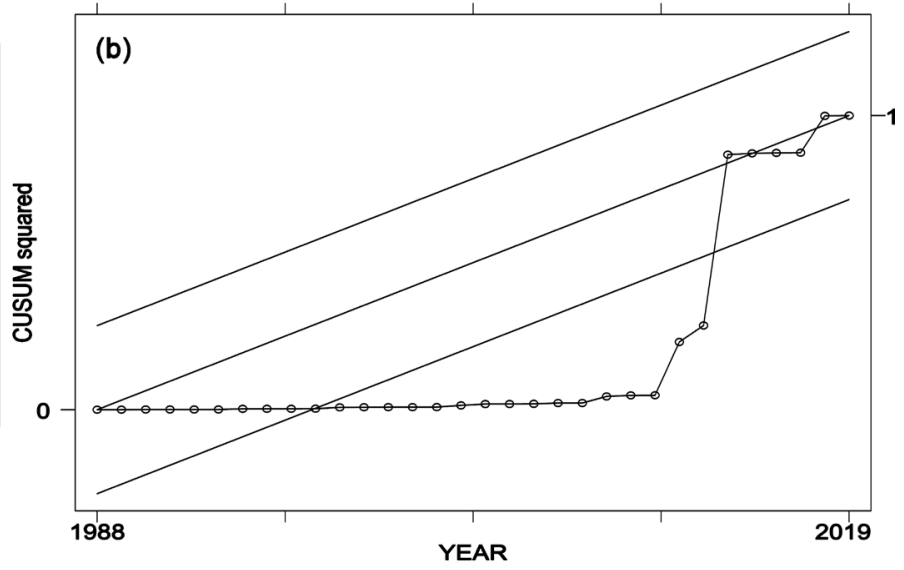

while the CUSUM squared test rejects the presence of stability in the money demand function.

Figure 11(a) and Figure 11(b) below reports the stability results from the CUSUM test and CUSUM squared test respectively for Guinea. Total stability is recorded in the money demand function based on the results from both the CUSUM squared test graph and the CUSUM graph. Both graphs are significant at 5\%.

Figure 12(a) and Figure 12(b) below reports the stability results from the CUSUM test and CUSUM squared test respectively for Ghana. Partial stability is recorded in the money demand function based on the CUSUM squared test graph while the CUSUM graph rejects the presence of stability in the money demand function.

Figure 13(a) and Figure 13(b) below reports the stability results from the CUSUM test and CUSUM squared test respectively for Ghana. Total stability is recorded in the money demand function based on the results from both the CUSUM squared test graph and the CUSUM graph. Both graphs are significant at $5 \%$. 

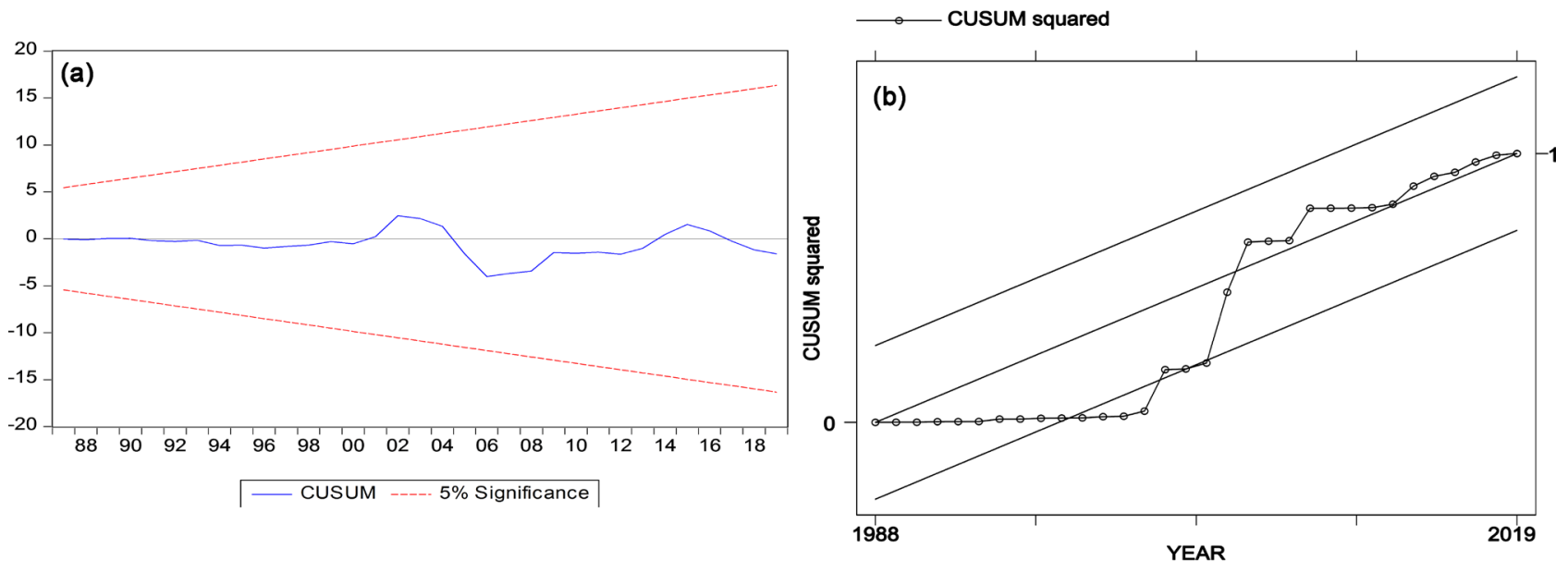

Figure 11. Stability graph of Guinea.
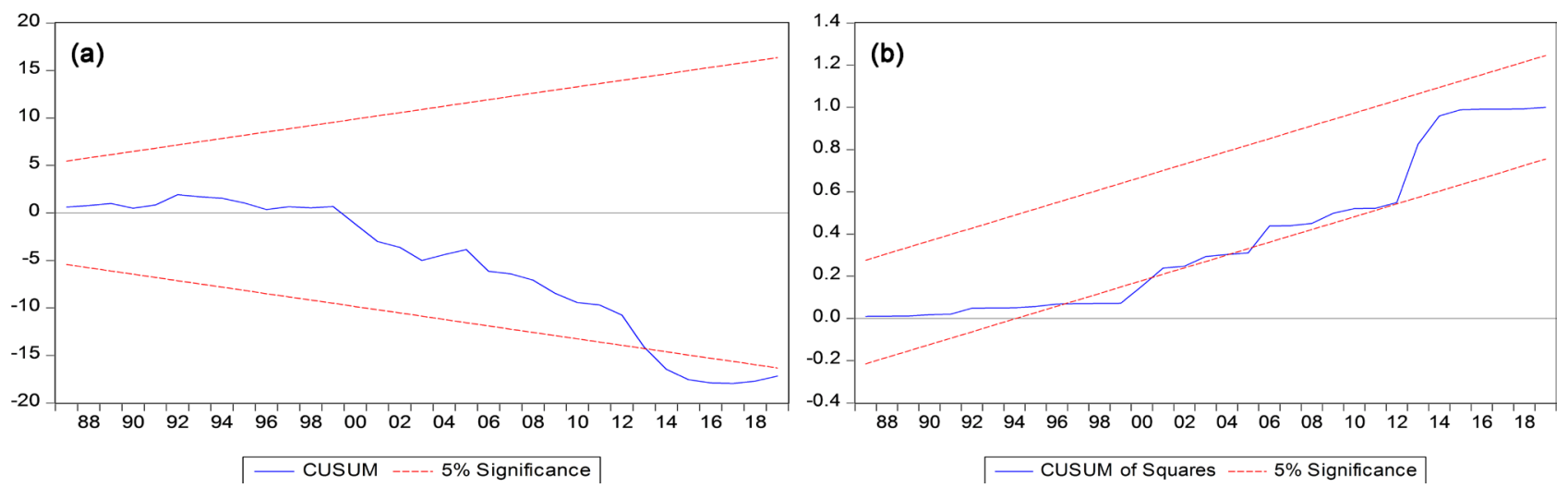

Figure 12. Stability graph of Ghana.
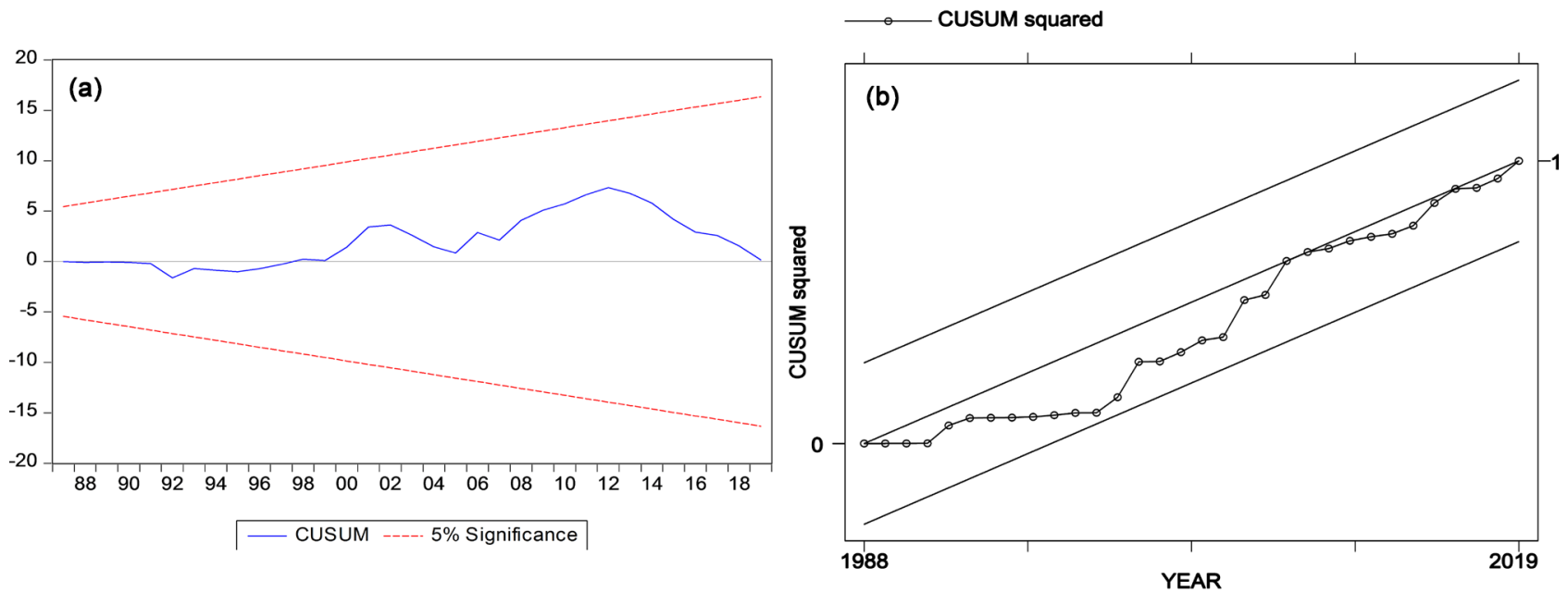

Figure 13. Stability graph of Gambia.

Figure 14(a) and Figure 14(b) below reports the stability results from the CUSUM test and CUSUM squared test respectively for Cape Verde. Partial stability is recorded in the money demand function based on the CUSUM graph while the CUSUM squared test rejects the presence of stability in the money 
demand function.

Figure 15(a) and Figure 15(b) below reports the stability results from the CUSUM test and CUSUM squared test respectively for Burkina Faso.Total stability is recorded in the money demand function based on the results from both the CUSUM squared test graph and the CUSUM graph. Both graphs are significant at $5 \%$.

Figure 16(a) and Figure 16(b) below reports the stability results from the CUSUM test and CUSUM squared test respectively for Benin. Partial stability is recorded in the money demand function based on the CUSUM graph while the CUSUM squared test rejects the presence of stability in the money demand function.

\section{Conclusions}

From the concerted effort among West African nations towards the formation of a monetary zone (adoption of a single currency), it is significant to draw the relevant lessons from both global financial meltdowns and the experiences of
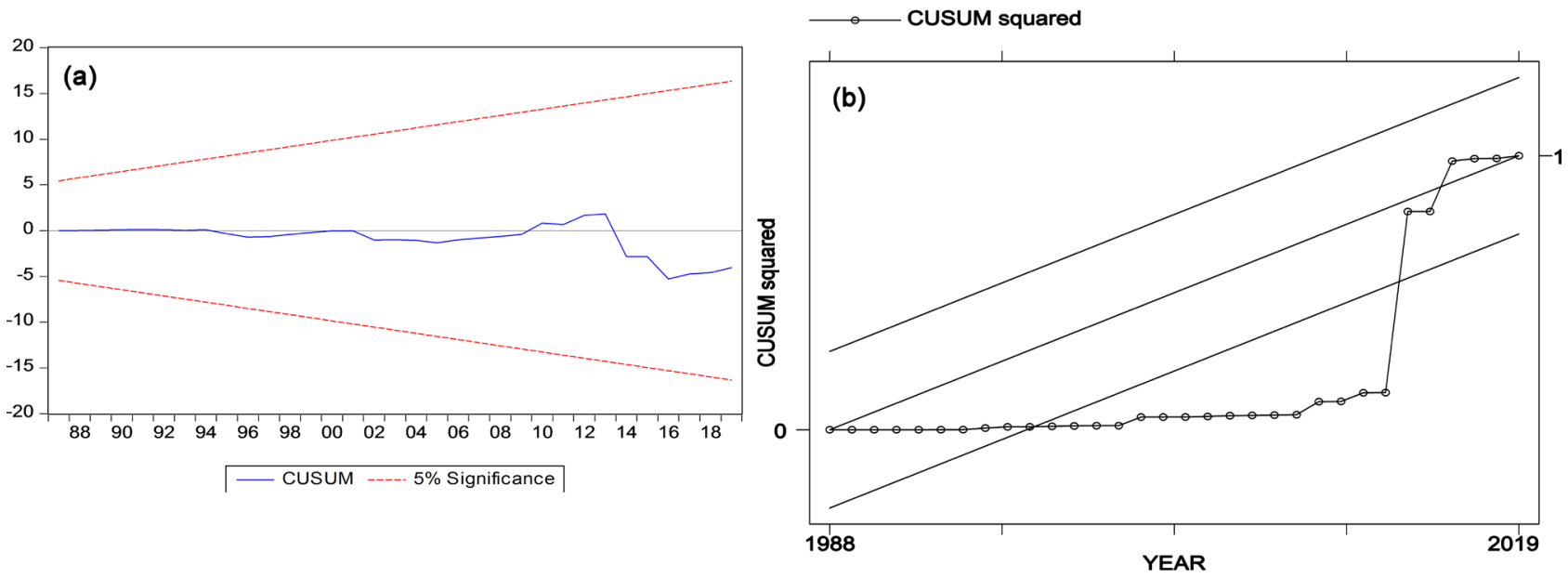

Figure 14. Stability graph of Cape Verde.
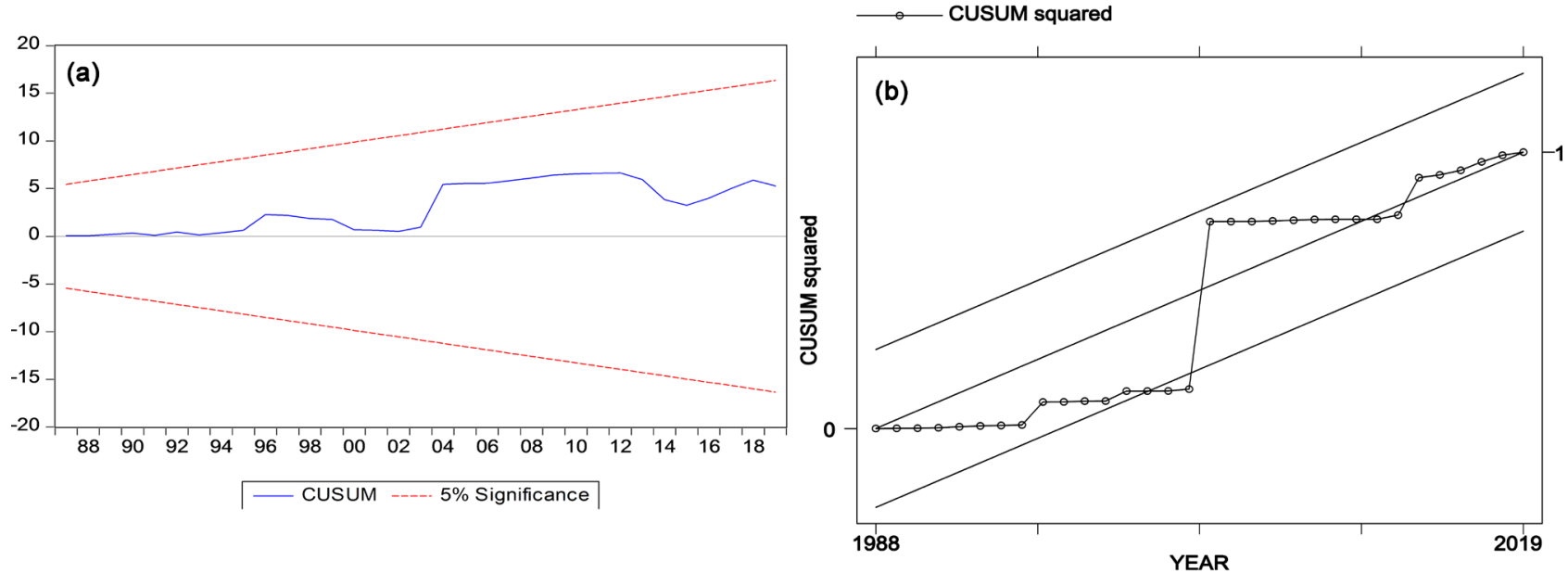

Figure 15. Stability graph of Burkina Faso. 

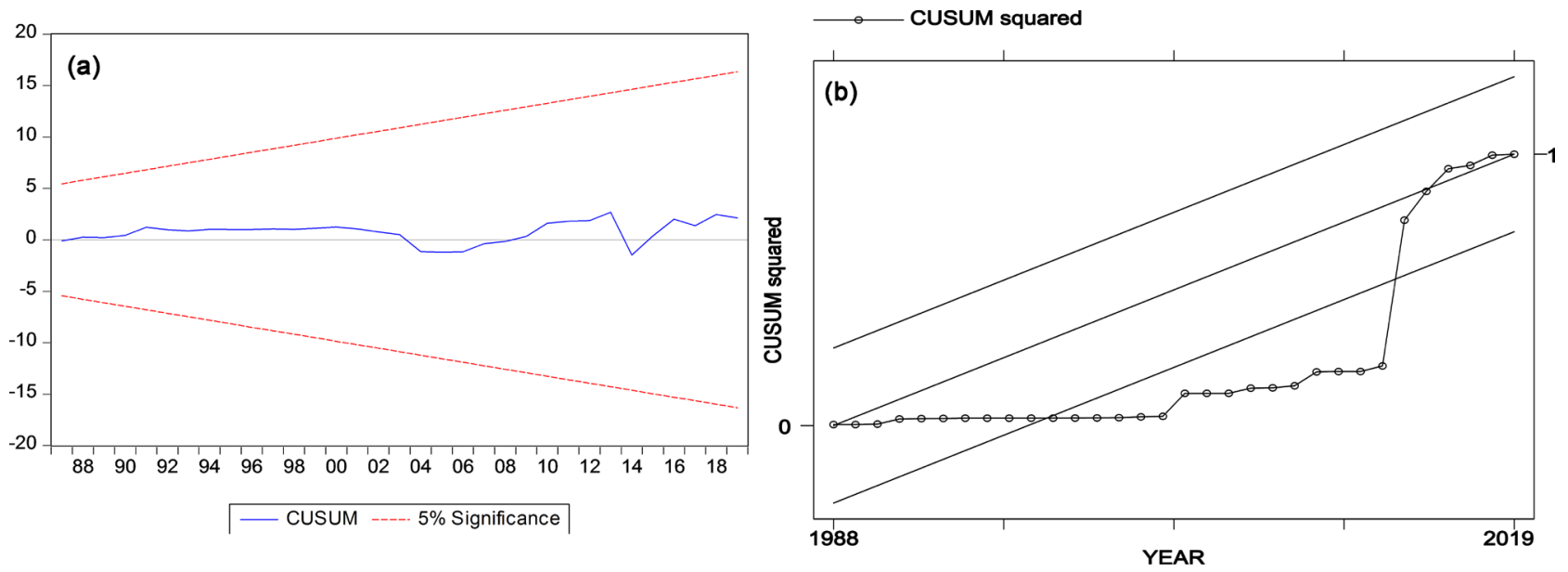

Figure 16. Stability graph of Benin.

other monetary zones such as the Euro zone. This is because the success of a monetary zone of any kind apparently depends on the stability and the long-run nexus between money demand and other macro-economic variables. It is also important to appreciate the fundamental difference among these countries to achieve a successful union. This is also necessary because in the event of a monetary union; each of the sixteen (16) countries forming part of the West Africa Monetary Zone will be abandoning their individual monetary policies in whole or partially and entrusting the responsibility to a centralized system or one central bank. The success of such a regime ultimately will depend on the stability of money demand across member countries.

This study records evidence of significant divergence in the stability of money demand across the sixteen (16) countries. The divergence is much conspicuous in both the CUSUM and CUSUM squared test. However, we only recorded co-integration for only Ghana. The money demand functions showed evidence of total stability for Gambia, Burkina Faso, Sierra Leon, Mauritania and Liberia based on the results from both the CUSMU and CUSUM squared test. Partial stability is also recorded in Senegal, Togo, Nigeria, Niger and Mali based on CUSUM test. The evidence from Ghana also points partial stability based on the CUSUM squared test.

Considering the fact that all the countries under consideration are in West Africa and are potential members of the West Africa Monetary Zone, the significant divergence in the stability of their money demand is of pivotal importance in designing monetary goals and targets to achieve the needed convergence for a monetary zone. These may include other things; the designing of country specific policies is to balance out all the heterogeneities causing the instabilities. Emphasis can be made on the biggest economy in West Africa (also Africa) - Nigeria, does not have a co-integrated money demand function and exhibits only partial stability. The same can be said about Ghana of partial stability but has a co-integrated money demand function. The case of Nigeria being the biggest economy among the sixteen (16) countries yet only partially stable and 
not co-integrated poses a significant threat to the proposed West Africa Monetary Zone. This finding is consistent with the findings of Asongu et al. (2019a) who found that from the proposed SAMU, South Africa being the largest economy did not have a co-integrated and an unstable money demand function.

The significant divergence and heterogeneity recorded in the stability of the money demand functions across the countries give ample credence to the potential complexities and ineffective that will characterize the implementation of monetary policies in the event of a monetary union in West Africa. This is to suggest that country specific features should not be overlooked in designing and implementation of a union. The findings of this study are consistent with the findings of Asongu et al. (2019b) and Harvey \& Cushing (2015) on their study of long-run stability of money demand in West Africa (using 13 countries) and the presence of a currency union in West Africa respectively.

\section{Suggestion for Further Study}

With the rapid growing and integration of financial markets in West Africa, it is suggested for further study, the inclusion of stock market performance and liquidity indictors to capture and explain some of the sources of the divergence and instability of the money demand functions of these countries.

\section{Conflicts of Interest}

The authors declare no conflicts of interest regarding the publication of this paper.

\section{References}

Asongu, S., Folarin, O., \& Biekpe, N. (2019a). The Stability of Demand for Money in the Proposed Southern African Monetary Union. International Journal of Emerging Markets, 15, 222-244. https://doi.org/10.1108/IJOEM-08-2018-0443

Asongu, S., Folarin, O., \& Biekpe, N. (2019b). The Long Run Stability of Money Demand in the Proposed West African Monetary Union. Research in International Business and Finance, 48, 483-495. https://doi.org/10.1016/i.ribaf.2018.11.001

Baharumshah, A. Z., Siti, H. M. A., \& Mansur, M. M. (2009). The Stability of Money Demand in China: Evidence from the ARDL Model. Economic Systems, 33, 231-244. https://doi.org/10.1016/j.ecosys.2009.06.001

Bahmani-Oskooee, M., \& Chi Wing Ng, R. (2002). Long-Run Demand for Money in Hong Kong: An Application of the ARDL Model. International Journal of Business and Economics, 1, 147-155.

Bahmani-Oskooee, M., \& Gelan, A. (2009). How Stable Is the Demand for Money in African Countries? Journal of Economic Studies, 36, 216-235. https://doi.org/10.1108/01443580910983825

Boone, L., Mikol, F., \& Van den Noord, P. (2004). Wealth Effects on Money Demand in EMU: Econometric Evidence. OECD Economics Department Working Papers 411.

Cassola, N., \& Morana, C. (2004). Monetary Policy and the Stock Market in the Euro Area. Journal of Policy Modeling, 26, 387-399. https://doi.org/10.1016/j.jpolmod.2004.03.012 
Darvas, Z. (2015). Does Money Matter in the Euro Area? Evidence from a New Divisia Index. Economics Letters, 133, 123-126. https://doi.org/10.1016/j.econlet.2015.05.034

Debrun, X., \& Masson, P. R. (2013). Modelling Monetary Union in Southern Africa: Welfare Evaluation for the CMA and SADC. South African Journal of Economics, 81, 275-291. https://doi.org/10.1111/saje.12008

Diop, S., Tillmann, P., \& Winker, P. (2017). A Monetary Stress Indicator for the Economic Community of West African States. Journal of African Development, 19, 1-18.

Dritsaki, C., \& Dritsaki, M. (2020). The Long-Run Money Demand Function: Empirical Evidence from Italy. International Journal of Economics and Financial Issues, 10, 186-195. https://doi.org/10.32479/ijefi.8943

Brown, R. L., Durbin, J., \& Evans, J. M. (1975). Techniques for Testing the Constancy of Regression Relationships over Time. Journal of the Royal Statistical Society: Series B (Methodological), 37, 149-163. https://doi.org/10.1111/j.2517-6161.1975.tb01532.x

Fleissig, A., \& Swofford, J. L. (1996). A Dynamic Asymptotically Ideal Model of Money Demand. Journal of Monetary Economics, 37, 371-380. https://doi.org/10.1016/0304-3932(96)01247-0

Folarin, O. E., \& Asongu, S. A. (2017). Financial Liberalization and Long-Run Stability of Money Demand in Nigeria. African Governance and Development Institute Working Paper No. 17/018, Yaoundé. https://doi.org/10.2139/ssrn.2979277

Foresti, P., \& Napolitano, O. (2014). Money Demand in the Eurozone: Do Monetary Aggregates Matter? Inzinerine Ekonomika Engineering Economics, 25, 497-503. https://doi.org/10.5755/j01.ee.25.5.3810

Harvey, S. K., \& Cushing, M. J. (2015). Is West African Monetary Zone (WAMZ) a Common Currency Area? Review of Development Finance, 5, 53-63. https://doi.org/10.1016/j.rdf.2015.05.001

Hamori, S., \& Tokihisa, A. (2001). Seasonal Cointegration and the Money Demand Function: Some Evidence from Japan. Applied Economics Letters, 8, 305-310. https://doi.org/10.1080/135048501750157468

Hossain, A. (1993). Financial Reforms, Stability of the Money Demand and Monetary Policy in Bangladesh: An Econometric Investigation. Indian Economic Review, 28, 85-100.

James, G. A. (2005). Money Demand and Financial Liberalization in Indonesia. Journal of Asian Economics, 16, 817-829. https://doi.org/10.1016/j.asieco.2005.08.003

Kumar, S. (2011). Financial Reforms and Money Demand: Evidence from 20 Developing Countries. Economic System, 35, 323-334. https://doi.org/10.1016/j.ecosys.2010.09.002

Rao, B. B., \& Kumar, S. (2009). A Panel Data Approach to the Demand for Money and the Effects of Financial Reforms in the Asian Countries. Economic Modelling, 26, 1012-1017. https://doi.org/10.1016/j.econmod.2009.03.008

Roohollah, Z., \& Azali, M. (2015). The Association between Aggregated and Disaggregated Stock Prices with Monetary Policy Using Asymmetric Cointegration and Error-Correction Modelling Approaches. Review of Development Finance, 5, 64-69. https://doi.org/10.1016/j.rdf.2014.07.002 